\title{
Flora and Annual Distribution of Flowers and Fruits in the Ubajara National Park, Ceará, Brazil
}

\author{
Andréa Pereira Silveira ${ }^{1}$ (D) 0000-0001-6785-5319 \\ Bruno Sousa de Menezes ${ }^{2}$ (D) 0000-0003-1134-8996 \\ Maria Iracema Bezerra Loiola ${ }^{2}$ (D) 0000-0003-3389-5560 \\ Luiz Wilson Lima-Verde ${ }^{2}$ (D) 0000-0002-6908-1692 \\ Dalva Neta e Zanina ${ }^{2}$ (D) 0000-0002-4653-445X \\ Ellen Cristina Dantas de Carvalho ${ }^{2}$ (D) 0000-0002-6887-3970 \\ Bruno Cruz de Souza ${ }^{2}$ (D) 0000-0002-4746-2638 \\ Rafael Carvalho da Costa ${ }^{2}$ (D) 0000-0002-0942-3128 \\ Waldir Mantovani² (D) 0000-0002-9394-6860 \\ Marcelo Oliveira Teles de Menezes ${ }^{3}$ (D) 0000-0003-2129-6843 \\ Lilian Maria Araújo Flores ${ }^{2}$ (D) 0000-0001-8037-6021 \\ Francisco Carlos Barboza Nogueira ${ }^{4}$ \\ Ligia Queiroz Matias² (D) 0000-0002-1889-5354 \\ Lívia Silvia Barbosa ${ }^{5}$ \\ Fernanda Melo Gomes ${ }^{2}$ (D) 0000-0002-4213-6577 \\ Luciana Silva Cordeiro $^{2}$ (D) 0000-0001-5624-2285 \\ Valéria da Silva Sampaio ${ }^{2}$ (D) 0000-0002-6551-8877 \\ Maria Edenilce Peixoto Batista ${ }^{2}$ (D) 0000-0002-1239-0902 \\ Raimundo Luciano Soares Neto ${ }^{6}$ (D) 0000-0002-5643-9464 \\ Maria Arlene Pessoa da Silva ${ }^{7}$ (D) 0000-0001-8148-5350 \\ Natália Barbosa Campos ${ }^{7}$ \\ Arycelle Alves de Oliveira ${ }^{7}$ \\ Francisca Soares de Araujo ${ }^{2}$ (D) 0000-0003-4661-6137
}

\begin{abstract}
Although the conservation of tropical biodiversity depends on protected areas, there is still a very large 'gap' of knowledge on the flora of Brazilian reserves, especially in the Northeast region of Brazil. Field and herbarium surveys of the phanerogamic flora of the Ubajara National Park, located on the Brazilian Northeast, were made and analyses on phenology and dispersal syndromes were performed. 418 taxa (213 trees and shrubs, 100 terrestrial herbs, 68 climbing plants, 33 sub-shrubs, two epiphytes, one hemiparasite and one aquatic herb) were recorded. The most representative families were: Fabaceae, Malvaceae, Asteraceae, Rubiaceae and Euphorbiaceae. The annual flowering / fruiting peak hypothesis was not fully confirmed, therefore, the forest may be an important food resource for the fauna all year long (especially in the moister region). Zoochory was the predominant dispersal syndrome in the moister area, whereas, autochory and anemochory together, predominated in the drier area.
\end{abstract}

Keywords: biodiversity, mountain forest, protected areas, evergreen forest, deciduous forest.

\footnotetext{
${ }^{1}$ Universidade Estadual do Ceará (UECE), Faculdade de Educação de Itapipoca (FACEDI), Itapipoca, CE, Brasil

${ }^{2}$ Universidade Federal do Ceará (UFC), Departamento de Biologia, Fortaleza, CE, Brasil

${ }^{3}$ Instituto Federal do Ceará (IFCE), Fortaleza, CE, Brasil

${ }^{4}$ Instituto Brasileiro do Meio Ambiente e dos Recursos Naturais Renováveis (IBAMA), Fortaleza, CE, Brasil

${ }^{5}$ Superintendência Estadual de Meio Ambiente (SEMACE), Fortaleza, CE, Brasil

${ }^{6}$ Universidade Federal de Pernambuco (UFPE), Departamento de Botânica, Recife, PE, Brasil

${ }^{7}$ Universidade Regional do Cariri (URCA), Departamento de Biologia, Crato, CE, Brasil
} 


\section{Introduction and objective}

The Brazilian semi-arid domain covers an area with a huge physiographic and climatic heterogeneity (associated with continentality, altitude and slope) - which influences moisture and rainfall (Mantovani et al., 2017). In this context, many kinds of vegetation may occur, such as stepic savanna (caatinga and carrasco), savanna (cerrado), coastal semideciduous forest (mata de tabuleiro), semideciduous/deciduous forest (mata seca) and semideciduous/evergreen forest (mata úmida) - the last two can be found mainly in montane regions (Souza \& Oliveira, 2006; IBGE, 2012; Moro et al., 2015; Mantovani et al., 2017). According to Zappi et al. (2015), at least 4,659 species of angiosperms were reported for all these plant formations.

The climate in montane regions of the Brazilian semi-arid domain is usually moister than the surroundings, due to orographic precipitation. These habitats are arranged like moist islands in a dry landscape, where forest flora and vegetation develop (Pereira et al., 2010; Nascimento et al., 2012; Pinto et al., 2012; Silva \& Figueiredo, 2013). Known as brejos de altitude, they often shelter deciduous, semideciduous and/or evergreen forests, with high species richness in comparison with the surrounding semi-arid landscapes (Pereira et al., 2010; Nascimento et al., 2012; Pinto et al., 2012; Silva \& Figueiredo, 2013). Thus, the brejos de altitude may form a patchy and fragmented mosaic of relic vegetation from a moist paleoclimate (Bétard et al., 2007; Mantovani et al., 2017).

The vegetation in the brejos de altitude is also an important resource to local and migratory fauna. The few researches made on these areas show that the seasonality and the water availability rule the phenological patterns and dispersion syndromes. It seems to be a tendency for this kind of biological community to have flower and fruit availabilities all year long (Locatelli $\&$ Machado, 2004). Also, it seems to be a predominance of zoochory in moist areas, while autochory and anemochory are predominant in dry areas (Diogo et al., 2016).

The Brazilian semi-arid domain, in which these montane forests are located, is also subjected to overpopulation, about $15 \%$ of the national population with 27 million inhabitants (Araújo, 2011), and to an intense rhythm of anthropic exploitation since the Portuguese colonization, especially on the highlands, which are moister than surrounding semi-arid depression (Lopes et al., 2017; Mantovani et al., 2017). Furthermore, protected areas in the Brazilian semi-arid domain account for only $7.8 \%$ of its area, of which only $1.3 \%$ is under the full protection usage regime, i.e. with restrictions to human intervention (Menezes et al., 2010). Thus, according to Rylands \& Brandon (2005), it is one of the most poorly protected regions of Brazil.

Although the conservation of tropical biodiversity depends on protected areas and their surrounding vegetation (Mantovani et al., 2017), there is still a very large 'gap' on the knowledge of the flora of Brazilian protected areas. Most of them are still poorly known, making a consistent analysis of their effectiveness for conservation very difficult (Oliveira et al., 2017; Mattar et al., 2018). According to Moro et al. (2015), floristic studies in the Ubajara mountain forest are highly desirable, since extensive floristic surveys for the Ceará highlands were only performed for Baturité and Araripe areas.

Thus, floristic surveys on Brazilian protected areas have a huge role on supporting conservation, management and restoration programs. Although many efforts have been made to assess the flora of Ceará (Loiola, 2013; Tabosa et al., 2016; Ribeiro \& Loiola, 2017; Carneiro et al., 2018; Sampaio et al., 2019), there are still very few published studies on the flora of the protected areas of the state of Ceará. From an inventory of the phanerogamic flora, it could be presented some analysis on the floristic composition, the phenological patterns and the dispersal syndromes found in the Ubajara National Park, located in a montane region of the state of Ceará, Brazil. This protected area is considered of extreme biological importance due to its high diversity and for being listed as a priority area for conservation and sustainable use (Brasil, 2007).

\section{Material and methods}

\subsection{Study area}

The Ubajara National Park is a full protection reserve, created in 1959. After two additions (the first in 1973 and the second in 2002) to the Ubajara National Park, its protected area reached 6,288 ha. It is located in the north of the Ibiapaba plateau, near the coast ( $\left.3^{\circ} 45^{\prime} \mathrm{S}, 40^{\circ} 54^{\prime} \mathrm{W}\right)$. Due to orographic rainfall, most part of the park is under a moister climate (Aw, Köppen-Geiger) than the surrounding valleys (Bsh, Köppen-Geiger). The terrain stands on a sedimentary sandstone on the higher altitude areas with outcrops of calcareous rocks on the windward slope. The Tropical Seasonal Deciduous Forest (TSDF) develops between 400-700 $\mathrm{m}$ and accounts for $72.1 \%$ of the area of the park. Between $700-900 \mathrm{~m}$, there is a Tropical Seasonal Evergreen Forest (TSEF), accounting for 18\% of the park; and in the lowlands (bellow $400 \mathrm{~m}$ ), there is the stepic savanna, which accounts for $9.9 \%$ of the park (Figure 1). The park is well preserved, it has about $88.7 \%$ of conserved vegetation and only $1.9 \%$ of disturbed vegetation. On the surroundings of the park, there is significant anthropogenic disturbance, especially on the moist side, with permeability of only $36.8 \%$, while on the dryer side the permeability is $56 \%$ (Mantovani et al., 2017).

On the lands with altitudes around 400-700 meters, the average annual rainfall is $950 \mathrm{~mm}$, concentrated from January to June, and the temperature is $28.2^{\circ} \mathrm{C}$ (Figure $2 \mathrm{~A}$ ). The soil is 


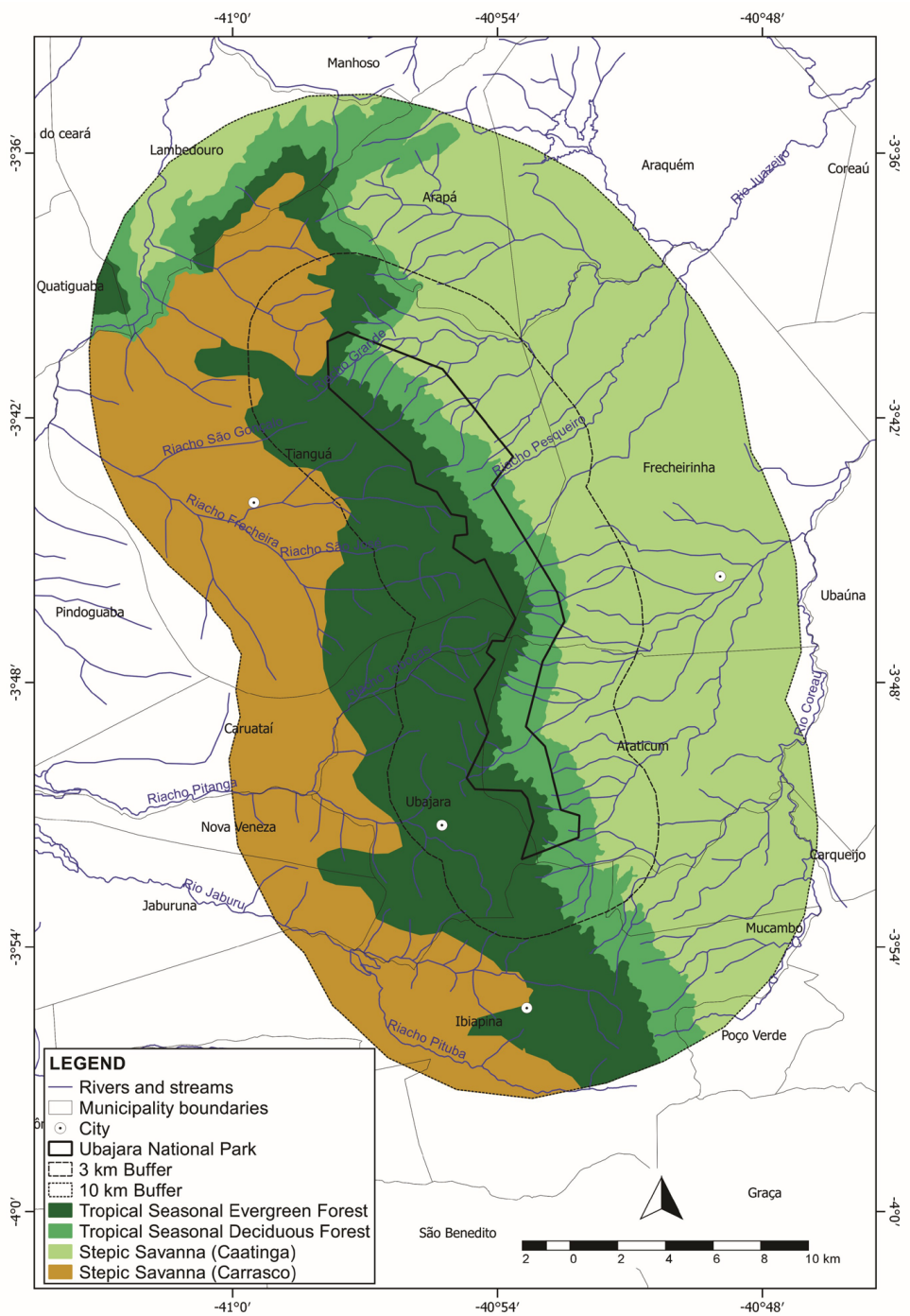

Figure 1. Vegetation of the Ubajara National Park in Ceara, Brazil.
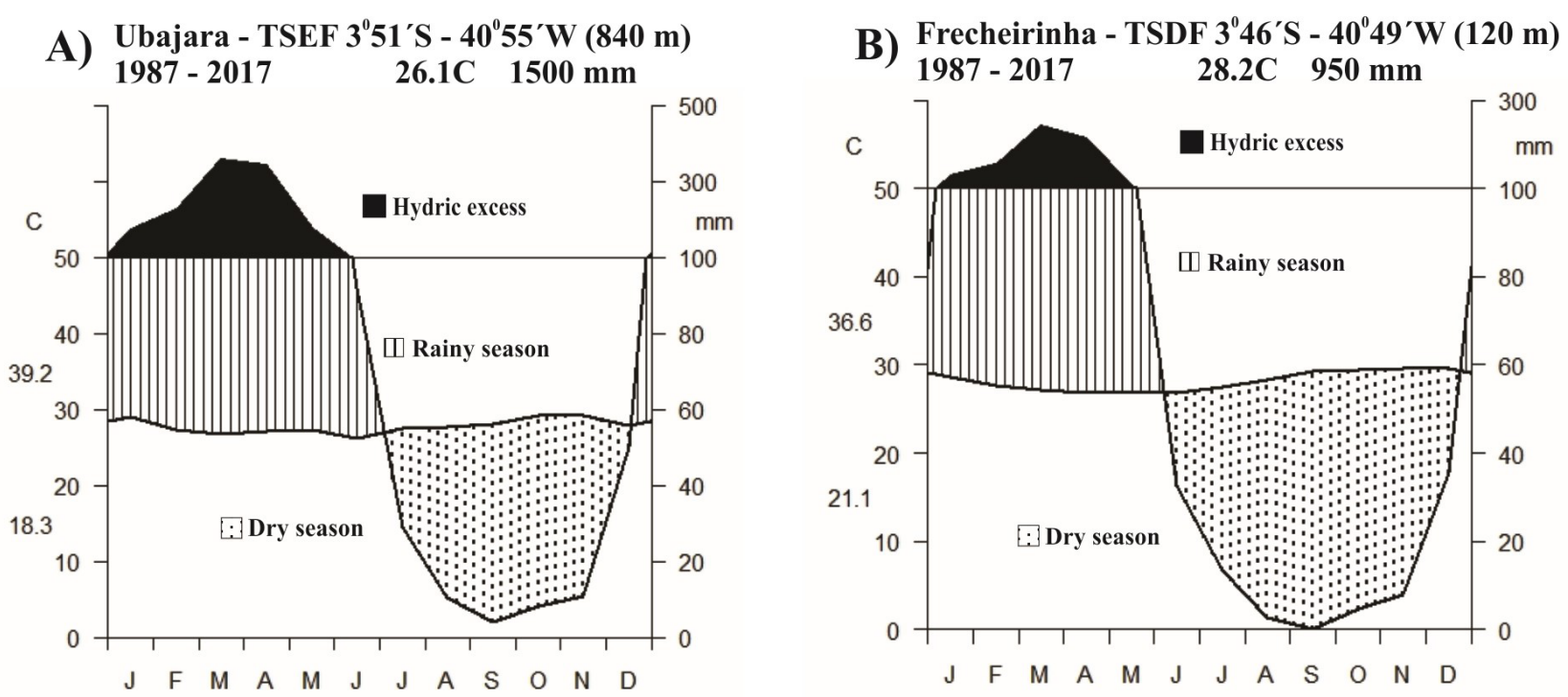

Figure 2. Walter \& Lieth climatic diagrams for Vegetation of the Ubajara National Park, Ceara, Brazil. (A) Tropical Seasonal Deciduous Forest (TSDF); and (B) Tropical Seasonal Evergreen Forest (TSEF). 
shallow and stony and could be classified as Dystrophic Regolith Neosol. The main headwaters of the Acaraú River basin are lotaced inside the Ubajara National Park. Additionally, the rainfall of the eastern slope of Ibiapaba infiltrates into calcareous rocks and resurge in the lower altitudes. This water is used to irrigate agriculture and also for household use. However, both the headwaters and the resurgent water can deplete during the dry season of deforested area (inside or outside the park) during consecutive years of drought.

The TSEF area (above $700 \mathrm{~m}$ ) exhibits average annual rainfall of $1,500 \mathrm{~mm}$, concentrated from January to June, and an average temperature of $26.1{ }^{\circ} \mathrm{C}$ (Figure 2B). The soil was classified as deep Dystrophic Tb Haplic Cambisol (CXBd).

\subsection{Data collection and analysis}

During approximately six years, the team went through the main park trails and vegetation patches to collect samples of the flora. The first collection was made between 2011 and 2014, being part of the project "Efetividade de UCs Federais do estado do Ceará na conservaçấo biológica do semiárido brasileiro" (CNPq/ICMBio 13/2011, No. 551998/2011-3). Additional samples were obtained between 2017 and 2018 during the project "Conservação da biodiversidade em nivel de paisagem: mudanças climáticas e distúrbios antropogênicos" (CNPq/ICMBio/FAPs 18/2017, No 421350/2017-2). Collections were also made in phytosociological and functional survey plots (unpublished data). Whenever possible, samples in reproductive state (with flowerbuds, flowers and/or fruits) were obtained. Vouchers were deposited in the Herbarium Prisco Bezerra (EAC) at the Universidade Federal do Ceará (UFC). The survey was complemented by gathering samples deposited by other researchers into the EAC collection.

The botanical determination was based on specialized literature, expert opinions and comparisons with other specimens from the EAC collection. The Angiosperm Phylogeny Group IV (APG IV, 2016) classification system was adopted. Names of the families, genera, species and authors names were confirmed on the International Plant Names Index (IPNI, 2019) and in the Flora of Brazil 2020 under construction website (JBRJ, 2019). The classification of exotic species was adopted, as suggested by Moro et al. (2012). It could be gathered data on family, species, vernacular name, main collector, plant formation, growth-form, flowering / fruiting period, fruit size, fruit type and dispersal syndrome only for native flora.

Classification of growth forms followed the Instituto Brasileiro de Geografia e Estatística (IBGE, 2012): tree - woody plants higher than $3 \mathrm{~m}$; shrub - woody plants with the main branch up to $50 \mathrm{~cm}$ above the ground level and generally shorter than $3 \mathrm{~m}$; sub-shrub - plants with woody main stem and secondary herbaceous branches, generally having a height of less than $2 \mathrm{~m}$; terrestrial herb - terrestrial plants with herbaceous aerial stem; epiphytic herb - plants with herbaceous stem which develop with another plant's support; climbing plants - lianas or vine plants with elongated stems, that usually are supported by a substrate or epiphytes that rely on their phorophyte for support, and also hemiparasite - plants that use the resources of their host, but have chlorophyll and perform photosynthesis.

The reproductive phenophases data were compiled of exsiccates of the EAC. Fruit size information and diaspore dispersal syndromes were obtained from herbarium sheets whenever possible, and from the literature when not. Diaspores were classified in small, medium and large following the length/width ratio criterion, as proposed by Tabarelli $\&$ Peres (2002). The dispersal syndromes were classified as anemochorous, autochorous or zoochorous, followed by Van der Pijl (1982).

The number of species per growth form, dispersal syndromes and diaspore size were analyzed through frequency diagrams. Flowering and fruiting patterns throughout the year were analyzed through circular histograms made with ORIANA 4 software (Kovach, 2011), based on the percentage of species which were flowering and fruiting (there was data on flowering for $80 \%$ of the species and on fruiting for only $30 \%$ ). The following parameters were calculated for the phenology evaluation (with months corresponding to $30^{\circ}$ angles): mean angle $(\mu$ ), representing the period of the year in which a particular phenophase occurred in most taxa; circular standard deviation (sd); Rayleigh (Z) test, which determines the significance of the mean angle; and vector $(r)$, which is a measure concentration around the mean angle. The null hypothesis on the $(\mathrm{Z})$ test is that flowering and fruiting are distributed evenly throughout the year, and therefore there is no seasonality of each phenophase. The alternative hypothesis is that there is seasonality (if the mean angle is significant). The vector $(r)$ varies from 0 (when the phenological activity is evenly distributed throughout the year) to 1 (when the phenological activity is seasonal/concentrated in a period of the year).

\section{Results and discussion}

Were found 418 specific/sub-specific taxa (84 families; 274 genera) could be found, from which 335 were found in the TSEF, 53 in TSDF, and 30 in both vegetations. Due to the absence of flowers or fruits, from this total number of registered data, only 22 species were identified up to genus and four were only identified up to family (Appendix A). This floristic richness was higher than any other ever recorded in 
montane forests of Brazilian semi-arid domain. According to the study of Nascimento et al. (2012), the researchers could find 293 species in Planalto da Borborema (Paraíba), nevertheless, Pereira et al. (2010) found 136 species in Serra Negra (Pernambuco), and Silva \& Figueiredo (2013) only 100 species in Serra da Meruoca (Ceará). However, this disparity in the species richness of the Ubajara National Park may be due to the high number of terrestrial herbs recorded, in other words, 100 species that comprise $23.9 \%$ of the species; on the other hand, other studies did not indicate no more than 10\% of herbs in the species richness. In addition, the other forests cited may have different areas and the respective surveys in each study may have different sampling effort, so the comparison may not be conclusive.

The families with the highest species richness were Fabaceae (64 spp.), Malvaceae (24 ssp.), Asteraceae (20 ssp.), Rubiaceae (18 spp.), Euphorbiaceae (16 spp.), Sapindaceae (14 spp.), Apocynaceae (13 spp.) and Myrtaceae (11 spp.). These data are in accordance to the same studies performed in other montane forests of the northeastern Brazil (Rodal \& Nascimento, 2002; Nascimento et al., 2012; Silveira et al., 2019). All other families had 10 or less species each. The most numerous genera were Erythroxylum (9 spp.), Passiflora (7 spp.), Croton (7 spp.) and Combretum (6 spp.) - Appendix A.

In addition to the species listed in the Appendix A, 28 exotic species ( 26 genera, 17 families) could be cataloged - Appendix B. The families with greatest numbers of exotic species were Araceaee (6 spp.), Apocynaceae and Amaryllidaceae (3 spp. each). These species are mostly ornamental and were planted on the park entrance and along the pathway to the cable car. Only five exotic species were found inside the park: Cryptostegia madagascariensis Bojer ex Decne., Coffea arabica L., Artocarpus heterophyllus Lam., Mangifera indica L. and Bambusa sp. In this case, the exotic species probably were present in the park before of its creation because it was a human occupied area. In the case of zoochorous plant species, exotic fruitful species may compete for the dispersers with native flora. Once the perpetuation of the native flora depends on both preservation and dispersal of diaspores (see Oliveira et al., 2017; Mattar et al., 2018), indigenous biodiversity may be somehow threatened.

Regarding the growth form, considering only indigenous species, it could be recorded 213 trees and shrubs, 100 terrestrial herbs, 68 climbing, 33 sub-shrubs, two epiphytic herbs, one hemiparasite and one aquatic herb (Figure 3A). Forests usually have a low proportion of herb richness in comparison to trees and shrubs. One possible cause of the high proportion of herbs in the flora of the Ubajara National Park may be a consequence of the compilation of data from the herbarium collection; while rainfall forest trees may exhibit supra-annual flowering/fruiting patterns (see Pereira et al., 2008), herbs usually have annual patterns (increasing the chances of being incorporated to the herbarium - once EAC have not accepted vegetative samples in the last years). A complete floristic checklist for the Ubajara National Park will only be possible after a long-term phytosociological survey or reproductive phenodinamic study on the future.

On spectrum of dispersal syndromes (Figure 3B), as expected, zoochory was predominant on the total flora $(55 \%)$ and in the TSEF alone (201 species, 56\%); while in the TSDF alone, the proportion of zoochory was lower (37 species, 46\%). This predominance of zoochory in forests was found by Locatelli \& Machado (2004) and by Diogo et al. (2016), showing that the Brazilian montane regions are important to preserve the richness of native species and available resource supply for local and migratory fauna. In both vegetations (TSEF and TSDF), the large and very large diaspores predominated, followed by medium and small ones (Figure 3C). The predominant type of fruit are capsule, drupe, bacca, legume, achene, samara, follicle and schizocarp (Figure 3D).

The Rayleigh test was only significant for fruit production (Figure 3F), seeing that this production is concentrated on June (in the end of the rainy season) with standard deviation from April to July ( $\mu=150.334^{\circ}$, sd $=105.854^{\circ}, Z=4.215$, $p=0.02, r=0.181)$. This fruiting peak in the beginning of the dry season seems to be an adaptation of plants from seasonal and dry climates, whose seeds have the following wet season to germinate and establish (Locatelli \& Machado; 2004; Pau et al., 2011). On the other hand, the vectors $r$ exhibited low results, indicating that the frequencies of the fruiting species are distributed evenly all year long. In addition, data on fruiting was available for only $30 \%$ of the species, so conclusions are not supported by these evidences. However, our results allowed generalizing that Ubajara National Park (as other montane forest from Northeast region of Brazil) offers resources for the fauna all year long.

Our results also showed that the Ubajara National Park has an important contribution to conservation of biodiversity, because the number of flowering plant species protected by this area is higher than those of other montane forests in Northeast region of Brazil (Carnaval et al., 2009; Homeier et al., 2010; Leite et al., 2016; Kamimura et al., 2017). The high diversity found in the Ubajara National Park may be attributed to its environmental heterogeneity, seeing that its topographic variation provides a range of different habitats, sheltering different vegetations and therefore a high floristic diversity. 


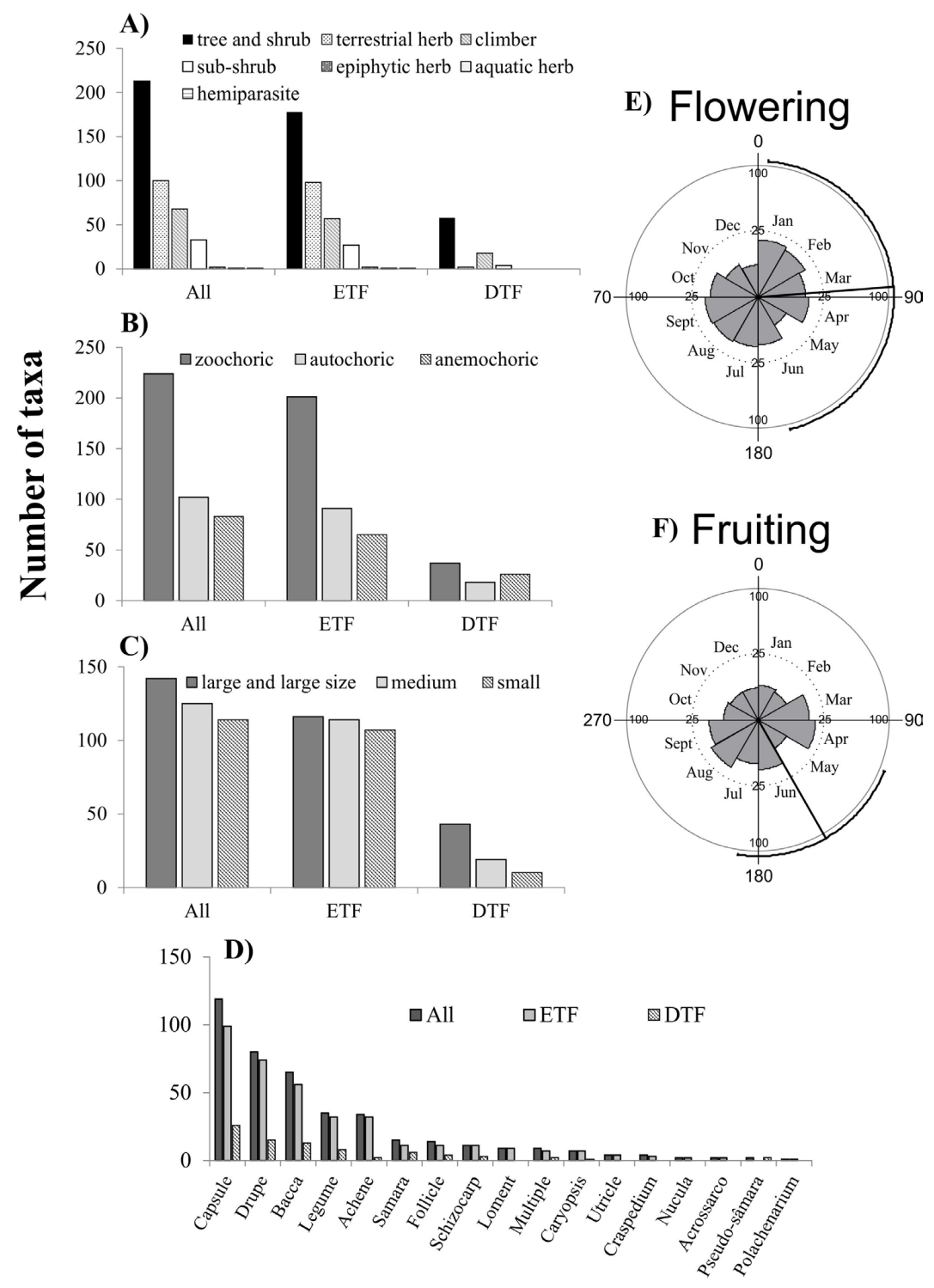

Figure 3. Number of taxa of the Evergreen Tropical Forest (ETF) and the Deciduous Tropical Forest (DTF) of the Ubajara National Park-Ceara, Brazil: (A) growth form; (B) dispersal syndrome; (C) fruit size; (D) fruit types; (E) circular histogram of relative flowering frequency $\left(\mu=85.524^{\circ}, \mathrm{sd}=167.128^{\circ}, \mathrm{Z}=0.031, p=0.97, r=0.014\right)$; and $(\mathrm{F})$ circular histogram of relative fruiting frequency $\left(\mu=150.334^{\circ}\right.$, $\left.\mathrm{sd}=105.854^{\circ}, \mathrm{Z}=4.215, p=0.02, r=0.181\right)$. The arrow shows the average date and its standard deviation.

\section{Conclusions}

The Ubajara National Park exhibits a huge species richness and an outstanding floristic composition - which makes this protected area very important for preservation of the local biodiversity. Beside the preservation of the plant species, the diversity of phenological patterns, types of fruits and dispersal syndromes create a substantial resource supply for local fauna almost all year long.

These results derived from a long-term sampling effort - an unparalleled study in the whole Brazilian semi-arid region. They represent not only an important contribution for the knowledge on the biodiversity of the semi-arid domain in northeast Brazil, but also a fundamental subside to the management of the park (including the formation of seed banks and seedlings production for restoration) and to further research on biodiversity, ecology, ethnobiology and effectiveness of protected areas.

Our results highlight the importance of studying the flora of the Brazilian protected areas. This is a fundamental step in assessing the effectiveness of protected areas in maintaining biodiversity.

\section{Acknowledgements}

We thank the Manegers of Ubajara PARNA for logistics support. 


\section{Submission status}

Received: 8 May, 2019

Accepted: 27 Dec., 2019

Associated Editor: Rodrigo Studart Corrêa (ORCID: 0000-00029422-2629)

\section{Correspondence to \\ Andréa Pereira Silveira}

Universidade Estadual do Ceará (UECE), Faculdade de Educação de Itapipoca (FACEDI), Av. da Universidade, S/N - Madalenas, CEP 62.205-090, Itapipoca, CE, Brasil

e-mail: andrea.silveira@uece.br

\section{Financial support}

Conselho Nacional de Desenvolvimento Científico e Tecnológico/ Instituto Chico Mendes de Conservação da Biodiversidade CNPq/ICMBio (551998/20113, 421350/2017-2) e Fundaçáo Cearense de Apoio ao Desenvolvimento Científico - FUNCAP (0132-00007.01.00/18).

\section{References}

Angiosperm Phylogeny Group IV - APG IV. An update of the Angiosperm Phylogeny Group classification for the orders and families of flowering plants. Botanical Journal of the Linnean Society 2016; 181(1): 1-20. http://dx.doi.org/10.1111/boj.12385.

Araújo SMS. A região semiárida do nordeste do Brasil: questões ambientais e possibilidades de uso sustentável dos recursos. Rios Eletrônica-Revista Científica da FASETE 2011; 5(5): 89-98.

Bétard F, Peulvast JP, Sales CV. Caracterização morfopedólogica de uma serra úmida no semi-árido do nordeste brasileiro: o caso do Maciço de Baturité-CE. Mercator-Revista de Geografia da UFC 2007; 6(12): 107-126.

Brasil. Ministério do Meio Ambiente. Áreas prioritárias para conservação, uso sustentável e repartição de benefícios da Biodiversidade Brasileira: Atualização - Portaria MMA n. ${ }^{\circ} 09$ de 23 de janeiro de 2007 [online]. Brasília: Ministério do Meio Ambiente; 2007 [cited 2019 Sept 18]. Available from: https://www.mma.gov. br/estruturas/chm/_arquivos/biodiversidade31.pdf

Carnaval AC, Hickerson MJ, Haddad CFB, Rodrigues MT, Moritz C. Stability predicts genetic diversity in the Brazilian Atlantic forest hotspot. Science 2009; 323(5915): 785-789. http://dx.doi.org/10.1126/ science.1166955. PMid:19197066.

Carneiro JAA, Rebouças NC, Ribeiro RTM, Gomes FM, Miranda RR, Soares-Neto RL et al. Flora do Ceará, Brasil: cleomaceae. Rodriguésia 2018; 69(4): 1659-1672. http://dx.doi.org/10.1590/21757860201869413 .

Diogo IJS, Martins FR, Verola CF, Costa IR. Variation in plantanimal interactions along an elevational gradient of moist forest in a semiarid area of Brazil. Acta Botanica Brasílica 2016; 30(1): 27-34. http://dx.doi.org/10.1590/0102-33062015abb0198.

Homeier J, Breckle SW, Günter S, Rollenbeck RT, Leuschner C. Tree diversity, forest structure and productivity along altitudinal and topographical gradients in a species-rich Ecuadorian montane rain forest. Biotropica 2010; 42(2): 140-148. http://dx.doi.org/10.1111/ j.1744-7429.2009.00547.x.
Instituto Brasileiro de Geografia e Estatística - IBGE. Manual técnico da vegetação brasileira. Rio de Janeiro: IBGE; 2012. 275 p.

International Plant Names Index - IPNI [online]. 2019 [cited 2019 Sept 18]. Available from: http://www.ipni.org

Jardim Botânico do Rio de Janeiro - JBRJ [online]. 2019 [cited 2019 Sept 18]. Available from: http://floradobrasil.jbrj.gov.br

Kamimura VA, Moraes PLR, Ribeiro HL, Joly CA, Assis MA. Tree diversity and elevational gradient: the case of Lauraceae in the Atlantic Rainforest. Flora 2017; 234(9): 84-91. http://dx.doi. org/10.1016/j.flora.2017.05.013.

Kovach WL. Oriana-circular statistics for windows, ver. 4. Wales, UK: Kovach Computing Services; 2011.

Leite YLR, Costa LP, Loss AC, Rocha RG, Batalha-Filho H, Bastos AC et al. Neotropical forest expansion during the last glacial period challenges refuge hypothesis. Proceedings of the National Academy of Sciences of the United States of America 2016; 113(4): 1008-1013. http://dx.doi.org/10.1073/pnas.1513062113. PMid:26755597.

Locatelli E, Machado IC. Fenologia das espécies arbóreas de uma mata serrana (Brejo de Altitude) em Pernambuco, Nordeste do Brasil. In: Porto KC, Cabral JJP, Tabarelli M, editors. Brejos de altitude em Pernambuco e Paraíba: história natural, ecologia e conservação. Brasília: Ministério do Meio Ambiente; 2004. p. 255-276.

Loiola MIB. A new species of Erythroxylum (Erythroxylaceae) from the Brazilian semiarid region. Phytotaxa 2013; 150(1): 61-64. http://dx.doi.org/10.11646/phytotaxa.150.1.5

Lopes SDF, Ramos MB, Almeida GRD. The role of mountains as refugia for biodiversity in brazilian caatinga: conservationist implications. Tropical Conservation Science 2017; 10: 1-12. http:// dx.doi.org/10.1177/1940082917702651.

Mantovani W, Anjos L, Monteiro RF, Araujo FS. A conservação da biodiversidade no domínio caatinga. Fortaleza: Edições UFC; 2017.

Mattar EPL, Barros TTV, Cunha BB, Souza JFD, Silva AMDC. Federal Conservation Units in Brazil: the situation of biomes and regions. Floresta e Ambiente 2018; 25(2): 2-10. http://dx.doi. org/10.1590/2179-8087.005115.

Menezes MOT, Araújo FS, Romero RE. O sistema de conservação biológica do estado do Ceará: diagnóstico e recomendações. Revista Eletrônica do Prodema 2010; 5(2): 7-31.

Moro MF, Macedo MB, Moura-Fé MM, Castro AS, Costa RC. Vegetação, unidades fitoecológicas e diversidade paisagística do estado do Ceará. Rodriguésia 2015; 66(3): 717-743. http://dx.doi. org/10.1590/2175-7860201566305.

Moro MF, Souza VC, Oliveira-Filho ATD, Queiroz LPD, Fraga $\mathrm{CND}$, Rodal MJN et al. Alienígenas na sala: o que fazer com espécies exóticas em trabalhos de taxonomia, florística e fitossociologia? Acta Botanica Brasílica 2012; 26(4): 991-999. http://dx.doi.org/10.1590/ S0102-33062012000400029.

Nascimento LM, Rodal MJN, Silva AG. Florística de uma floresta estacional no Planalto da Borborema, nordeste do Brasil. Rodriguésia 2012; 63(2): 429-440. http://dx.doi.org/10.1590/S217578602012000200015 .

Oliveira U, Soares-Filho BS, Paglia AP, Brescovit AD, Carvalho $\mathrm{CJB}$, Silva DP et al. Biodiversity conservation gaps in the Brazilian 
protected areas. Scientific Reports 2017; 7(1): 9141. http://dx.doi. org/10.1038/s41598-017-08707-2. PMid:28831073.

Pau S, Wolkovich EM, Cook BI, Davies TJ, Kraft NJB, Bolmgren $\mathrm{K}$ et al. Predicting phenology by integrating ecology, evolution and climate science. Global Change Biology 2011; 17(12): 3633-3643. http://dx.doi.org/10.1111/j.1365-2486.2011.02515.x.

Pereira RCA, Silva JA, Barbosa JIS. Flora de um "brejo-de-altitude" de Pernambuco: reserva Ecológica da Serra Negra. Anais da Academia Pernambucana de Ciência Agronômica 2010; 7: 286-304.

Pereira TS, Costa MLMN, Moraes LFD, Luchiari C. Fenologia de espécies arbóreas em floresta Atlântica da Reserva Biológica de Poço das Antas, Rio de Janeiro, Brasil. Iheringia. Série Botânica 2008; 63(2): 329-339.

Pinto MSC, Sampaio EVSB, Nascimento LM. Florística e estrutura da vegetação de um brejo de altitude em Pesqueira, PE, Brasil. Revista Nordestina de Biologia 2012; 21(1): 47-79.

Ribeiro RTM, Loiola MIB. Flora do Ceará, Brasil: bixaceae. Rodriguésia 2017; 68(4): 1313-1322. http://dx.doi.org/10.1590/21757860201768413.

Rodal MJ, Nascimento LM. Levantamento florístico da floresta serrana da reserva biológica de Serra Negra, microrregião de Itaparica, Pernambuco, Brasil. Acta Botanica Brasílica 2002; 16(4): 481-500. http://dx.doi.org/10.1590/S0102-33062002000400009.

Rylands AB, Brandon K. Unidades de conservação brasileiras. Megadiversidade 2005; 1(1): 27-35.
Sampaio VS, Vieira IMF, Lima-Júnior EAL, Loiola MIB. Flora do Ceará, Brasil: Solanum (Solanaceae). Rodriguésia 2019; 70: e02512017. http://dx.doi.org/10.1590/2175-7860201970029.

Silva MEA, Figueiredo MF. Flora fanerogâmica de um enclave úmido no Ceará: serra da Meruoca. Enciclopédia Biosfera 2013; 9(17): 2811-2820.

Silveira AP, Loiola MIB, Gomes VS, Verde LWL, Oliveira TS, Silva EF et al. Flora of Baturite - Ceara: a wet island in the brazilian semiarid. Floresta e Ambiente 2019. In press.

Souza MJN, Oliveira VPV. Os enclaves úmidos e sub-úmidos do semi-árido do nordeste brasileiro. Revista Mercator 2006; 5(9): 85-102.

Tabarelli M, Peres CA. Abiotic and vertebrate seed dispersal in the Brazilian Atlantic forest: implications for forest regeneration. Biological Conservation 2002; 106(2): 165-176. http://dx.doi. org/10.1016/S0006-3207(01)00243-9.

Tabosa FRS, Almeida EM, Melo E, Loiola MIB. Flora do Ceará, Brasil: polygonaceae. Rodriguésia 2016; 67(4): 981-996. http:// dx.doi.org/10.1590/2175-7860201667409.

Van der Pijl L. Principles of dispersal in higher plants. Berlim: Springer-Verlag. 1982. http://dx.doi.org/10.1007/978-3-642-87925-8.

Zappi DC, Filardi FLR, Leitman P, Souza VC, Walter BMT, Pirani JR et al. Growing knowledge: an overview of seed plant diversity in Brazil. Rodriguésia 2015; 66(4): 1085-1113. http://dx.doi. org/10.1590/2175-7860201566411. 
Appendix A. List of families and species of the Tropical Seasonal Deciduous Forest (TSDF) and the Tropical Seasonal Evergreen Forest (TSEF) of the Ubajara National Park in Ceará, Brazil, where $\mathrm{x}=$ presence of taxa.

\begin{tabular}{|c|c|c|c|}
\hline \multirow{2}{*}{ FAMILY / SPECIES } & \multicolumn{2}{|c|}{ VEGETATION } & \multirow{2}{*}{ MAIN COLLECTOR } \\
\hline & TSDF & TSEF & \\
\hline \multicolumn{4}{|l|}{ 1. Acanthaceae } \\
\hline Ruellia asperula (Mart. ex Ness) Lindau & & $\mathrm{x}$ & Fernandes, A. (EAC 27690) \\
\hline R. geminiflora Kunth & & $\mathrm{x}$ & Cavalcanti, F.S. 549 \\
\hline \multicolumn{4}{|l|}{ 2. Alstromeriaceae } \\
\hline Bomarea edulis (Tussac) Herb. & & $\mathrm{x}$ & Loiola, M.I.B. 1591 \\
\hline \multicolumn{4}{|l|}{ 3. Amaranthaceae } \\
\hline Alternanthera brasiliana (L.) Kuntze & & $\mathrm{x}$ & Castro, A.J. (EAC 6153) \\
\hline A. tenella Colla & & $\mathrm{x}$ & Loiola, M.I.B. 1528 \\
\hline Cyathula achyranthoides (Kunth) Moq. & & $\mathrm{x}$ & Fernandes, A. (EAC 3973) \\
\hline Iresine diffusa Humb. \& Bonpl. ex Willd. & & $\mathrm{x}$ & Fernandes, A. (EAC 5064) \\
\hline \multicolumn{4}{|l|}{ 4. Amaryllidaceae } \\
\hline Hippeastrum stylosum Herb. & & $\mathrm{x}$ & Cavalcanti, F.S. 595 \\
\hline Zephyranthes cearensis (Herb.) Baker & & $\mathrm{x}$ & Cavalcanti, F.S. 594 \\
\hline \multicolumn{4}{|l|}{ 5. Anacardiaceae } \\
\hline Astronium fraxinifolium Schott & $\mathrm{x}$ & & Fernandes, A. (EAC 15095) \\
\hline Myracrodruon urundeuva Allemão & $\mathrm{x}$ & & Matos (EAC 6897) \\
\hline Spondias mombin L. & $\mathrm{x}$ & & Freitas, B. \\
\hline Tapirira guianensis Aubl. & & $\mathrm{x}$ & Loiola, M.I.B. 1940 \\
\hline Thyrsodium spruceanum Benth. & & $\mathrm{x}$ & Fernandes, A. (EAC 27839) \\
\hline \multicolumn{4}{|l|}{ 6. Annonaceae } \\
\hline Annona exsucca DC. & & $\mathrm{x}$ & Fernandes, A. (EAC 27751) \\
\hline A. leptopetala (R.E.Fr.) H.Rainer & & $\mathrm{x}$ & Fernandes, A. (EAC 8287) \\
\hline Annonaceae sp1 & & $\mathrm{x}$ & Sterile material \\
\hline Guatteria pogonopus Mart. & & $\mathrm{x}$ & Loiola, M.I.B. 1939 \\
\hline G. schomburgkiana Mart. & & $\mathrm{x}$ & Lima-Verde, L.W. 3542 \\
\hline Guatteria sp. & & $\mathrm{x}$ & Sterile material \\
\hline Oxandra sessiliflora R.E.Fr. & & $\mathrm{x}$ & Loiola, M.I.B. 1489 \\
\hline Xylopia sp. & $\mathrm{x}$ & & Sterile material \\
\hline \multicolumn{4}{|l|}{ 7. Apiaceae } \\
\hline Spananthe paniculata Jacq. & & $\mathrm{x}$ & Fernandes, A. (EAC 27943) \\
\hline \multicolumn{4}{|l|}{ 8. Apocynaceae } \\
\hline Aspidosperma pyrifolium Mart. \& Zucc. & $\mathrm{x}$ & & Loiola, M.I.B. 1872 \\
\hline A. riedelii subsp. oliganthum (Woodson) Marc.-Ferr. & $\mathrm{x}$ & & Fernandes, A. (EAC 8895) \\
\hline A. subincanum Mart. & $\mathrm{x}$ & & Araújo, F.S. 699 \\
\hline Forsteronia pubescens A. DC. & & $\mathrm{x}$ & Loiola, M.I.B. 2229 \\
\hline Mandevilla hirsuta (A.Rich.) K.Schum. & & $\mathrm{x}$ & Fernandes, A. (EAC 27684) \\
\hline Matelea denticulata (Vahl) Fontella \& E.A.Schwarz & & $\mathrm{x}$ & Loiola, M.I.B. 1527 \\
\hline Rauvolfia ligustrina Willd. & & $\mathrm{x}$ & Fernandes, A. (EAC 27923) \\
\hline R. paucifolia A.DC. & & $\mathrm{x}$ & Castro, A.S.J. 1377 \\
\hline Schubertia grandiflora Mart. & & $\mathrm{x}$ & Fernandes, A. (EAC 27886) \\
\hline Tabernaemontana catharinensis A.DC. & & $\mathrm{x}$ & Fernandes, A. (EAC 3997) \\
\hline Apocynaceae sp 1 & & $\mathrm{x}$ & Sterile material \\
\hline Apocynaceae sp2 & & $\mathrm{x}$ & Sterile material \\
\hline Apocynaceae sp3 & & $\mathrm{x}$ & Sterile material \\
\hline \multicolumn{4}{|l|}{ 9. Araceae } \\
\hline Anthurium sinuatum Benth. ex Schott & & $\mathrm{x}$ & Fernandes, A. (EAC 27781) \\
\hline Caladium bicolor (Aiton) Vent. & & $\mathrm{x}$ & Fernandes, A. (EAC 28493) \\
\hline Monstera praetermissa E.G.Gonç. \& Temponi & & $\mathrm{x}$ & Loiola, M.I.B. 1804 \\
\hline Philodendron acutatum Schott & & $\mathrm{x}$ & Fernandes, A. (EAC 27815) \\
\hline P. ornatum Schott & & $\mathrm{x}$ & Fernandes, A. (EAC 28491) \\
\hline
\end{tabular}


Appendix A. Continued...

\begin{tabular}{|c|c|c|c|}
\hline \multirow{2}{*}{ FAMILY / SPECIES } & \multicolumn{2}{|c|}{ VEGETATION } & \multirow{2}{*}{ MAIN COLLECTOR } \\
\hline & TSDF & TSEF & \\
\hline Spathicarpa gardneri Schott & & $\mathrm{x}$ & Andrade, I.M. (EAC 28492) \\
\hline Taccarum ulei Engl. \& K.Krause & & $\mathrm{x}$ & Loiola, M.I.B. 2215 \\
\hline \multicolumn{4}{|l|}{ 10. Arecaceae } \\
\hline Acrocomia aculeata (Jacq.) Lodd. ex Mart. & & $\mathrm{x}$ & Observed \\
\hline Attalea speciosa Mart. ex Spreng. & & $\mathrm{x}$ & Balick, M.J. 1354 \\
\hline \multicolumn{4}{|l|}{ 11. Asteraceae } \\
\hline Acanthospermum australe (Loefl.) Kuntze & & $\mathrm{x}$ & Fernandes, A. (EAC 2948) \\
\hline Acmella uliginosa (Sw.) Cass. & & $\mathrm{x}$ & Matias, L.Q. 677 \\
\hline Ageratum conyzoides $\mathrm{L}$. & & $\mathrm{x}$ & Fernandes, A. (EAC 3940) \\
\hline Aspilia andrade-limae J.U.Santos & & $\mathrm{x}$ & Fernandes, A. (EAC 5066) \\
\hline Blainvillea acmella (L.) Philipson & & $\mathrm{x}$ & Fernandes, A. (EAC 3937) \\
\hline Bidens cynapiifolia Kunth & & $\mathrm{x}$ & Castro, A.J. (EAC 6152) \\
\hline B. pilosa L. & & $\mathrm{x}$ & Fernandes, A. (EAC 16657) \\
\hline Brickellia diffusa (Vahl) A.Gray & & $\mathrm{x}$ & Fernandes, A. (EAC 4008) \\
\hline Centratherum punctatum Camss. & & $\mathrm{x}$ & Fernandes, A. (EAC 3993) \\
\hline Conocliniopsis prasiifolia (DC.) R.M.King \& H.Rob. & & $\mathrm{x}$ & Fernandes, A. (EAC 3899) \\
\hline Elephantopus mollis Kunth & & $\mathrm{x}$ & Fernandes, A. (EAC 5083) \\
\hline Emilia fosbergii Nicolson & & $\mathrm{x}$ & Loiola, M.I.B. 1523 \\
\hline E. sonchifolia (L.) DC. ex Wight & & $\mathrm{x}$ & Fernandes, A. (EAC 27850) \\
\hline Galinsoga parviflora Cav. & & $\mathrm{x}$ & Loiola, M.I.B. 1511 \\
\hline Gymnanthemum amygdalinum (Delile) Sch.Bip. ex Walp. & & $\mathrm{x}$ & Fernandes, A. (EAC 27478) \\
\hline Lepidaploa salzmannii (DC.) H.Rob. & & $\mathrm{x}$ & Fernandes, A. (EAC 3887) \\
\hline Sphagneticola trilobata (L.) Pruski & & $\mathrm{x}$ & Alvesm M. 421 \\
\hline Pseudogynoxys cabrerae H.Rob. \& Cuatrec & & $\mathrm{x}$ & Loiola, M.I.B. 1506 \\
\hline Trixis antimenorrhoea (Schrank) Kuntze & & $\mathrm{x}$ & Fernandes, A. (EAC 27768) \\
\hline Wedelia villosa Gardner & & $\mathrm{x}$ & Fernades, A. (EAC 27803) \\
\hline \multicolumn{4}{|l|}{ 12. Begoniaceae } \\
\hline Begonia reniformis Dryand. & & $\mathrm{x}$ & Fernandes, A. (EAC 3983) \\
\hline B. saxicola A.DC. & & $\mathrm{x}$ & Fernandes, A. (EAC 27773) \\
\hline \multicolumn{4}{|l|}{ 13. Bignoniaceae } \\
\hline Anemopaegma citrinum Mart. ex DC. & & $\mathrm{x}$ & Andrade, I.M. (EAC 28494) \\
\hline Bignonia sp. & & $\mathrm{x}$ & Fernandes, A. (EAC 27788) \\
\hline Dolichandra ungüis-cati (L.) L.G.Lohmann & & $\mathrm{x}$ & Fernandes, A. (EAC 27948) \\
\hline Fridericia dispar (Bureau ex K.Schum.) L.G.Lohmann & $\mathrm{x}$ & & Loiola, M.I.B. 1837 \\
\hline F. platyphylla (Cham.) L.G.Lohmann & $\mathrm{x}$ & & Sterile material \\
\hline F. triplinervea (Mart. ex DC.) L.G.Lohmann & $\mathrm{x}$ & & Sterile material \\
\hline Handroanthus impetiginosus (Mart. ex DC.) Mattos & $\mathrm{x}$ & $\mathrm{x}$ & Sterile material \\
\hline H. serratifolium (A.H.Genter) S. Grose & $\mathrm{x}$ & $\mathrm{x}$ & Sterile material \\
\hline Zeyheria tuberculosa (Vell.) Bureau ex Verl. & & $\mathrm{x}$ & Fernandes, A. (EAC 27955) \\
\hline \multicolumn{4}{|l|}{ 14. Bixaceae } \\
\hline Cochlospermum vitifolium (Willd.) Spreng. & $\mathrm{x}$ & & Sterile material \\
\hline \multicolumn{4}{|l|}{ 15. Boraginaceae } \\
\hline Cordia bicolor A.DC. & & $\mathrm{x}$ & Loiola, M.I.B. 1919 \\
\hline C. rufescens A.DC. & & $\mathrm{x}$ & Sterile material \\
\hline C. toqueve Aubl. & $\mathrm{x}$ & $\mathrm{x}$ & Fernandes, A. (EAC 27816) \\
\hline C. trichotoma (Vell.) Arráb. ex Steud. & & $\mathrm{x}$ & Loiola, M.I.B. 1820 \\
\hline Euploca procumbens (Mill.) Diane \& Hilger & & $\mathrm{x}$ & Fernandes, A. (EAC 27855) \\
\hline Tournefortia membranacea (Gardner) DC. & & $\mathrm{x}$ & Fernandes, A. (EAC 27761) \\
\hline Varronia leucomalloides (Taroda) J.S.Mill. & & $\mathrm{x}$ & Fernandes, A. (EAC 8282) \\
\hline V. polycephala Lam. & & $\mathrm{x}$ & Soares Neto, R.L. 34 \\
\hline \multicolumn{4}{|l|}{ 16. Burseraceae } \\
\hline Protium heptaphyllum (Aubl.) Marchand & & $\mathrm{x}$ & Loiola, M.I.B. 1922 \\
\hline
\end{tabular}


Appendix A. Continued...

\begin{tabular}{|c|c|c|c|}
\hline \multirow{2}{*}{ FAMILY / SPECIES } & \multicolumn{2}{|c|}{ VEGETATION } & \multirow{2}{*}{ MAIN COLLECTOR } \\
\hline & TSDF & TSEF & \\
\hline P. warmingianum Marchand & & $\mathrm{x}$ & Mata, M.F. (EAC 15587) \\
\hline \multicolumn{4}{|l|}{ 17. Cactaceae } \\
\hline Cereus jamacaru DC. & $\mathrm{x}$ & & Observed \\
\hline Epiphyllum phyllanthus (L.) Haw. & & $\mathrm{x}$ & Loiola, M.I.B. 2202 \\
\hline \multicolumn{4}{|l|}{ 18. Campanulaceae } \\
\hline Centropogon cornutus (L.) Druce & & $\mathrm{x}$ & Fernandes, A. (EAC 5090) \\
\hline \multicolumn{4}{|l|}{ 19. Cannabaceae } \\
\hline Celtis brasiliensis (Gardner) Planch. & & $\mathrm{x}$ & Fernandes, A. (EAC 27793) \\
\hline C. iguanaea (Jacq.) Sarg. & & $\mathrm{x}$ & Loiola, M.I.B. 1822 \\
\hline Trema micrantha (L.) Blume & & $\mathrm{x}$ & Nunes, E. (EAC 27875) \\
\hline \multicolumn{4}{|l|}{ 20. Capparaceae } \\
\hline Cynophalla flexuosa (L.) J. Presl & $\mathrm{x}$ & & Fernandes, A. (EAC 27673) \\
\hline C. hastata (Jacq.) J.Presl & & $\mathrm{x}$ & Fernandes, A. (EAC 27991) \\
\hline \multicolumn{4}{|l|}{ 21. Chrysobalanaceae } \\
\hline Hirtella glandulosa Spreng. & & $\mathrm{x}$ & Fernandes, A. (EAC 3970) \\
\hline \multicolumn{4}{|l|}{ 22. Clusiaceae } \\
\hline Clusia pana-panari (Aubl.) Choisy & & $\mathrm{x}$ & Fernandes, A. (EAC 27766) \\
\hline \multicolumn{4}{|l|}{ 23. Combretaceae } \\
\hline Buchenavia tetraphylla (Aubl.) R.A.Howard. & & $\mathrm{x}$ & Fernandes, A. (EAC 27750) \\
\hline Combretum duarteanum Cambess. & & $\mathrm{x}$ & Fernandes, A. (EAC 4002) \\
\hline C. fruticosum (Loefl.) Stuntz & $\mathrm{x}$ & & Loiola, M.I.B. 1845 \\
\hline C. glaucocarpum Mart. & $\mathrm{x}$ & $\mathrm{x}$ & Loiola, M.I.B. 2219 \\
\hline C. lanceolatum Pohl ex Eichler & & $\mathrm{x}$ & Loiola, M.I.B. (EAC 53995) \\
\hline C. laxum Jacq. & & $\mathrm{x}$ & Cavalcanti, F.S. 551 \\
\hline C. leprosum Mart. & $\mathrm{x}$ & $\mathrm{x}$ & Nunes, E. (EAC 27829) \\
\hline \multicolumn{4}{|l|}{ 24. Commelinaceae } \\
\hline Dichorisandra hexandra (Aubl.) Kuntze ex Hand-Mazz. & & $\mathrm{x}$ & Loiola, M.I.B. 2273 \\
\hline Floscopa glabrata (Kunth) Hassk. & & $\mathrm{x}$ & Cavalcanti, F.S. 550 \\
\hline \multicolumn{4}{|l|}{ 25. Convolvulaceae } \\
\hline $\begin{array}{l}\text { Distimake macrocalyx (Ruiz \& Pav.) A.R. Simões \& } \\
\text { Staples }\end{array}$ & & $\mathrm{x}$ & Fernandes, A. (EAC 27495) \\
\hline Ipomoea alba L. & $\mathrm{x}$ & $\mathrm{x}$ & Cavalcanti, F.S. (EAC 28459) \\
\hline I. blanchetii Choisy & & $\mathrm{x}$ & Fernandes, A. (EAC 27907) \\
\hline I. brasiliana (Choisy) Meisn. & $\mathrm{x}$ & & Araújo, F.S. 602 \\
\hline I. marcellia Meisn. & $\mathrm{x}$ & & Sterile material \\
\hline Merremia umbellata (L.) Hallier f. & & $\mathrm{x}$ & Observed \\
\hline \multicolumn{4}{|l|}{ 26. Cucurbitaceae } \\
\hline Cayaponia tayuya (Vell.) Cogn. & $\mathrm{x}$ & $\mathrm{x}$ & Cavalcanti, F.S. 554 \\
\hline Psiguria ternata (M.Roem.) C.Jeffrey & & $\mathrm{x}$ & Loiola, M.I.B. 2263 \\
\hline P. umbrosa (Kunth) C.Jeffrey & & $\mathrm{x}$ & Fernandes, A. (EAC 27884) \\
\hline Rytidostylis amazonica (Mart. ex Cogn.) Kuntze & & $\mathrm{x}$ & Loiola, M.I.B. 2244 \\
\hline \multicolumn{4}{|l|}{ 27. Cyperaceae } \\
\hline Cyperus laxus Lam. & & $\mathrm{x}$ & Loiola, M.I.B. 1545 \\
\hline C. odoratus L. & & $\mathrm{x}$ & Loiola, M.I.B. 1790 \\
\hline C. simplex Kunth & & $\mathrm{x}$ & Loiola, M.I.B. 1797 \\
\hline Fuirena umbellata Rottb. & & $\mathrm{x}$ & Fernandes, A. (EAC 5073) \\
\hline Rhynchospora corymbosa (L.) Britton & & $\mathrm{x}$ & Fernandes, A. (EAC 27882) \\
\hline Scleria latifolia Sw. & & $\mathrm{x}$ & Fernandes, A. (EAC 3978) \\
\hline S. secans (L.) Urb. & & $\mathrm{x}$ & Loiola, M.I.B. 1490 \\
\hline \multicolumn{4}{|l|}{ 28. Dilleniaceae } \\
\hline Doliocarpus dentatus (Aubl.) Standl. & & $\mathrm{x}$ & Cavalcanti, F.S. (EAC 28474) \\
\hline
\end{tabular}


Appendix A. Continued...

FAMILY / SPECIES

\section{Dioscoreaceae}

Dioscorea dodecaneura Vell.

D. hassleriana Chodat

D. multiflora Mart. ex Griseb

D. orthogoneura Uline ex Hochr.

D. piperifolia Humb. \& Bonpl. ex Willd.

\section{Ebenaceae}

Diospyros inconstans subsp. obovata (Mart. ex Miq.)

B.Walln.

D. sericea A.DC.

\section{Elaeocarpaceae}

Sloanea obtusa (Splitg.) Schum.

32. Erythroxylaceae

Erythroxylum barbatum O.E.Schulz

E. citrifolium A.St.-Hil.

E. deciduum A.St.-Hil.

E. laetevirens O.E.Schulz

E. mucronatum Benth.

E. pulchrum A.St.-Hil.

E. simonis Plowman

E. subglaucescens Peyr.

E. subrotundum A.St.-Hil.

\section{Euphorbiaceae}

Acalypha poiretii Spreng.

A. villosa Jacq.

Cnidoscolus urens (L.) Arthur

Croton adenocalyx Baill.

C. betaceus Baill.

C. blanchetianus Baill.

C. floribundus Spreng.

C. hirtus L'Hér.

C. jacobinensis Baill.

C. triqueter Lam.

Dalechampia pernambucensis Baill.

D. tiliifolia Lam.

Joannesia princeps Vell.

Margaritaria nobilis L.f.

Microstachys corniculata (Vahl) Griseb.

Tragia cearensis Pax \& K.Hoffm.

\section{Fabaceae}

Aeschynomene paniculata Willd. ex Vogel

A. sensitiva Sw.

Albizia polycephala (Benth.) Killip ex Record

Anadenanthera colubrina var. cebil (Griseb.) Altschul

Ancistrotropis peduncularis (Kunth) A.Delgado

Andira vermifuga Mart. ex Benth.

Apuleia leiocarpa (Vogel) J.F.Macbr.

Ateleia guaraya Herzog

Bauhinia pulchella Benth.

B. ungulata L. var. ungulata

Bowdichia virgilioides Kunth

Calopogonium mucunoides Desv.
VEGETATION

TSDF TSEF

\section{MAIN COLLECTOR}

Sterile material

Fernandes, A. (EAC 28016)

Loiola, M.I.B. 1547

Fernandes, A. (EAC 5003)

Fernandes, A. (EAC 8239)

$\mathrm{x}$

Fernandes, A. (EAC 5096)

Fernandes, A. (EAC 27869)

$\mathrm{x}$

Fernandes, A. (EAC 8264)

Fernandes, A. (EAC 27852)

Loiola, M.I.B. 1778

Loiola, M.I.B. 2231

Araújo, F.S. 827

Loiola, M.I.B. 2255

Castro, A.S.F. 1378

Loiola, M.I.B. 2211

Fernandes, A. (EAC 27910)

Costa-Lima, J.L. 2263

$\mathrm{X}$

Fernandes, A. (EAC 27936)

Nunes, E. (EAC 27830)

Fernandes, A. (EAC 27716)

Nunes, E. (EAC 27876)

Loiola, M.I.B. 2180

Loiola, M.I.B. 2183

Fernandes, A. (EAC 27925)

Fernandes, A, (EAC 27927)

Loiola, M.I.B. 2214

Loiola, M.I.B. 2200

Fernandes, A. (EAC 27952)

Fernandes, A. (EAC 15081)

Loiola, M.I.B. 2233

Loiola, M.I.B. 1590

Fernandes, A. (EAC 27935)

Loiola, M.I.B. 1475

Fernandes, A. (EAC 8241)

Fernandes, A. (EAC 3949) Loiola, M.I.B. 1938

Fernandes, A. (EAC 27782)

Fernandes, A. (EAC 3930)

Fernandes, A. (EAC 7947)

Fernandes, A. (EAC 4094)

Fernandes, A. (EAC 3957)

Loiola, M.I.B. 1978

Loiola, M.I.B. 1947

Fernandes, A. (EAC 3936)

Fernandes, A. (EAC 4012) 
Appendix A. Continued...

\section{FAMILY / SPECIES}

C. velutinum (Benth.) Amshoff

Canavalia grandiflora Benth.

Cassia ferruginea (Schrad.) Schrad. ex DC.

Centrosema brasilianum (L.) Benth. var. brasilianum

C. sagittatum (Humb. \& Bonpl. Ex Willd.) Brandegee

Chamaecrista duckeana (P.Bezerra \& Afr.Fern.) H.S.Irwin

\& Barneby

Chamaecrista rotundifolia (Pers.) Greene

Copaifera duckei Dwyer

Crotalaria incana L.

C. retusa $\mathrm{L}$.

C. stipularia Desv.

Dalbergia cearensis Ducke

Deguelia nitidula (Benth.) A.M.G.Azevedo \& R.A.

Camargo

Desmodium axillare (Sw.) DC.

D. barbatum (L.) Benth.

D. distortum (Aubl.) J.F.Macbr.

D. incanum (Sw.) DC.

D. uncinatum (Jacq.) DC.

Dioclea megacarpa Rolfe

D. sclerocarpa Ducke

D. virgata (Rich.) Amshoff

Galactia striata (Jacq.) Urb.

Hymenaea coubaril L.

Indigofera suffruticosa Mill.

Inga alba (Sw.) Willd.

I. ingoides (Rich.) Willd.

Libidibia ferrea (Mart. ex Tul.) L.P.Queiroz var. ferrea

Machaerium acutifolium Vogel

M. amplum Benth.

M. hirtum (Vell.) Stellfeld

Mimosa caesalpiniifolia Benth.

M. candollei R. Grether

M. paraibana Barneby

M. xanthocentra Mart.

Mucuna sloanei Fawc. \& Rendle

Myroxylon peruiferum L.f.

Ormosia arborea (Vell.) Harms

Periandra coccinea (Schrader) Benth.

Phanera glabra (Jacq.) Vaz

P. outimouta (Aubl.) L.P.Queiroz

Pterocarpus sp.

Pterogyne nitens Tul.

Rhynchosia phaseoloides (Sw.) DC.

Senegalia polyphylla (DC.) Britton \& Rose

S. tenuifolia (L.) Britton \& Rose

Senna pendula var. dolichandra H.S.Irwin \& Barneby

S. obtusifolia (L.) H.S.Irwin \& Barneby

S. pilifera (Vogel) H.S.Irwin \& Barneby

S. rizzinii H.S.Irwin \& Barneby

Stryphnodendron guianense (Aubl.) Benth.
VEGETATION

TSDF TSEF

$\mathrm{x}$

$\mathrm{X}$

$\mathrm{X}$

$\mathrm{x}$

$\mathrm{X}$

$\mathrm{X}$

$\mathrm{X}$

$\mathrm{X}$

$\mathrm{X}$

$\mathrm{X}$

$\mathrm{X}$

$\mathrm{x}$

$\mathrm{x}$

$\mathrm{X}$

$\mathrm{x}$

$\mathrm{x}$

$\mathrm{X}$

$\mathrm{X}$

$\mathrm{X}$

$\mathrm{X}$

$\mathrm{X}$

$\mathrm{X}$

$\mathrm{X}$

$\mathrm{X}$

$\mathrm{X}$

$\mathrm{x}$

$\mathrm{x}$

$\mathrm{X}$

$\mathrm{X}$

$\mathrm{X}$

$\mathrm{X}$

$\mathrm{X}$

$\mathrm{x}$

$\mathrm{X}$
MAIN COLLECTOR

Fernandes, A. (EAC 27497)

Loiola, M.I.B. 1935

Loiola, M.I.B. 2234

Fernandes, A. (EAC 3929)

Loiola, M.I.B. 1486

Fernandes, A. (EAC 27853)

Fernandes, A. (EAC 27990)

Loiola, M.I.B. 1895

Fernandes, A. (EAC 3964)

Fernandes, A. (EAC 3984)

Fernandes, A. (EAC 3948)

Fernandes, A. (EAC 3935)

Araújo, F.S. (UEC 96442)

Loiola, M.I.B. 1488

Fernandes, A. (EAC 3913)

Fernandes, A. (EAC 3900)

Fernandes, A. (EAC 27749)

Fernandes, A. (EAC 3934)

Loiola, M.I.B. 1864

Loiola, M.I.B. 1805

Cavalcanti, F.S. 560

Fernandes, A. (EAC 4009)

Loiola, M.I.B. 1926

Cavalcanti, F.S. (EAC 28463)

Loiola, M.I.B. 1921

Loiola, M.I.B. 1829

Loiola, M.I.B. 1846

Cavalcanti, F.S. 534

Loiola, M.I.B. 1827

Loiola, M.I.B. 1930

Loiola, M.I.B. 2191

Fernandes, A. (EAC 27893)

Fernandes, A. (EAC 3962)

Fernandes, A. (EAC 3908)

Loiola, M.I.B. (EAC 52593)

Fernandes, A. (EAC 10788)

Fernandes, A. (EAC 27778)

Loiola, M.I.B. 1815

Loiola, M.I.B. 1928

Andrade, M. (EAC 28711)

Sterile material

Fernandes, A. (EAC 3938)

Fernandes, A. (EAC 15585)

Loiola, M.I.B. 1931

Loiola, M.I.B. 1544

Fernandes, A. (EAC 3906)

Fernandes, A. (EAC 27921)

Fernandes, A. (EAC 4000)

Cavalcanti, F.S. 539

Loiola, M.I.B. 1936 
Appendix A. Continued...

\begin{tabular}{|c|c|c|c|}
\hline \multirow{2}{*}{ FAMILY / SPECIES } & \multicolumn{2}{|c|}{ VEGETATION } & \multirow{2}{*}{ MAIN COLLECTOR } \\
\hline & TSDF & TSEF & \\
\hline Stylosanthes capitata Vogel & & $\mathrm{x}$ & Fernandes, A. (EAC 3958) \\
\hline S. scabra Vogel & & $\mathrm{x}$ & Fernandes, A. (EAC 3965) \\
\hline \multicolumn{4}{|l|}{ 35. Gesneriaceae } \\
\hline Drymonia serrulata (Jacq.) Mart. & & $\mathrm{x}$ & Castro, A.S.F. (EAC 24892) \\
\hline Sinningia nordestina Chautems, Baracho \& Siqueira-Filho & & $\mathrm{x}$ & Loiola, M.I.B. 1812 \\
\hline $\begin{array}{l}\text { Sphaerorrhiza sarmentiana (Gardner ex Hook) Roalson } \\
\text { \& Boggan }\end{array}$ & & $\mathrm{x}$ & Fernandes et al. (EAC 28014) \\
\hline \multicolumn{4}{|l|}{ 36. Heliconiaceae } \\
\hline Heliconia psittacorum L.f. & & $\mathrm{x}$ & Loiola, M.I.B. 1780 \\
\hline H. spathocircinata Aristeg. & & $\mathrm{x}$ & Fernandes, A. (EAC 3971) \\
\hline \multicolumn{4}{|l|}{ 37. Hernandiaceae } \\
\hline Sparattanthelium botocudorum Mart. & & $\mathrm{x}$ & Loiola, M.I.B. 2242 \\
\hline \multicolumn{4}{|l|}{ 38. Hypericaceae } \\
\hline Vismia guianensis (Aubl.) Choisy & & $\mathrm{x}$ & Fernandes, A. (EAC 27762) \\
\hline \multicolumn{4}{|l|}{ 39. Iridaceae } \\
\hline Eleutherine bulbosa (Mill.) Urb. & & $\mathrm{x}$ & Nunes, E. (EAC 27872) \\
\hline \multicolumn{4}{|l|}{ 40. Lamiaceae } \\
\hline Hyptis atrorubens Poit. & & $\mathrm{x}$ & Fernandes, A. (EAC 3967) \\
\hline Cantinoa mutabilis (Rich.) Harley \& J.F.B. Pastore & & $\mathrm{x}$ & Fernandes, A. (EAC 3979) \\
\hline Marsypianthes chamaedrys (Vahl) Kuntze & & $\mathrm{x}$ & Fernandes, A. (EAC 27890) \\
\hline Ocimum gratissimum $\mathrm{L}$. & & $\mathrm{x}$ & Fernandes, A. (EAC 3992) \\
\hline Vitex panshiniana Moldenke & & $\mathrm{x}$ & Fernandes, A. (EAC 14839) \\
\hline \multicolumn{4}{|l|}{ 41. Lauraceae } \\
\hline Aiouea saligna Meissn. & & $\mathrm{x}$ & Sterile material \\
\hline Ocotea glomerata (Nees) Mez & & $\mathrm{x}$ & Fernandes, A. (EAC 28013) \\
\hline Ocotea sp. & & $\mathrm{x}$ & Sterile material \\
\hline \multicolumn{4}{|l|}{ 42. Lythraceae } \\
\hline Cuphea impatientifolia A. St.-Hil. & $\mathrm{x}$ & & Fernandes, A. (EAC 27912) \\
\hline \multicolumn{4}{|l|}{ 43. Loganiaceae } \\
\hline Strychnos sp. & $\mathrm{x}$ & & Sterile material \\
\hline \multicolumn{4}{|l|}{ 44. Malpighiaceae } \\
\hline Banisteriopsis stellaris (Griseb.) B. Gates & $\mathrm{x}$ & & Sterile material \\
\hline Bunchosia pernambucana W.R.Anderson & & $\mathrm{x}$ & Fernandes, A. (EAC 27801) \\
\hline Byrsonima crispa A.Juss. & & $\mathrm{x}$ & Loiola, M.I.B. 1933 \\
\hline B. sericea DC. & & $\mathrm{x}$ & Fernandes, A. (EAC 3914) \\
\hline Byrsonima sp. & & $\mathrm{x}$ & Sterile material \\
\hline $\begin{array}{l}\text { Diplopterys pubipetala (A.Juss.) W.R. Anderson \& C. } \\
\text { Davis }\end{array}$ & & $\mathrm{x}$ & Fernandes, A. (EAC 27736) \\
\hline Heteropterys coeloptera A.Juss. & $\mathrm{x}$ & & Sterile material \\
\hline H. macrodena (DC.) W.R.Anderson & $\mathrm{x}$ & $\mathrm{x}$ & Fernandes, A. (EAC 7940) \\
\hline Janusia anisandra (A.Juss.) Griseb & $\mathrm{x}$ & & Sterile material \\
\hline \multicolumn{4}{|l|}{ 45. Malvaceae } \\
\hline Apeiba tibourbou Aubl. & & $\mathrm{x}$ & Loiola, M.I.B. 2224 \\
\hline Briquetiastrum spicatum (Kunth) Bovini & & $\mathrm{x}$ & Fernandes, A. (EAC 4007) \\
\hline Byttneria catalpifolia Jacq. & & $\mathrm{x}$ & Loiola, M.I.B. 2265 \\
\hline Callianthe bezerrae (Monteiro) Donnel & & $\mathrm{x}$ & Fernandes, A. (EAC 31063) \\
\hline Ceiba glaziovii (Kuntze) K. Schum. & $\mathrm{x}$ & & Observed \\
\hline Guazuma ulmifolia Lam. & $\mathrm{x}$ & $\mathrm{x}$ & Loiola, M.I.B. 1542 \\
\hline Helicteres heptandra L.B.Sm. & $\mathrm{x}$ & $\mathrm{x}$ & Loiola, M.I.B. 2220 \\
\hline Luehea sp. & $\mathrm{x}$ & & Sterile material \\
\hline Melochia nodiflora Sw. & & $\mathrm{x}$ & Fernandes, A. (EAC 4003) \\
\hline Pavonia cancellata (L.) Cav. & & $\mathrm{x}$ & Lima-Verde, L.W. 2390 \\
\hline
\end{tabular}


Appendix A. Continued...

\begin{tabular}{|c|c|c|c|}
\hline \multirow{2}{*}{ FAMILY / SPECIES } & \multicolumn{2}{|c|}{ VEGETATION } & \multirow{2}{*}{ MAIN COLLECTOR } \\
\hline & TSDF & TSEF & \\
\hline P. malacophylla (Link \& Otto) Garcke & & $\mathrm{x}$ & Loiola, M.I.B. 1929 \\
\hline Peltaea trinervis (C.Presl) Krapov. \& Cristóbal & & $\mathrm{x}$ & Fernandes, A. (EAC 5074) \\
\hline $\begin{array}{l}\text { Pseudobombax marginatum (A.St.-Hil., Juss. \& Cambess.) } \\
\text { A. Robyns }\end{array}$ & $\mathrm{x}$ & & Sterile material \\
\hline Sida acuta Burm.f. & $\mathrm{x}$ & & Fernandes, A. (EAC 27937) \\
\hline Sida cordifolia $\mathrm{L}$. & & $\mathrm{x}$ & Fernandes, A. (EAC 27693) \\
\hline S. glutinosa Comm. ex Cav. & & $\mathrm{x}$ & Fernandes, A. (EAC 4195) \\
\hline S. rhombifolia L. & & $\mathrm{x}$ & Fernandes, A. (EAC 27894) \\
\hline S. urens L. & & $\mathrm{x}$ & Mata, M.F. (EAC 15599) \\
\hline Sidastrum micranthum (A.St.-Hil.) Fryxell & & $\mathrm{x}$ & Fernandes, A. (EAC 5091) \\
\hline Sterculia striata A.St.-Hil. \& Naudin & $\mathrm{x}$ & & Sterile material \\
\hline Triumfetta semitriloba Jacq. & & $\mathrm{x}$ & Cavalcanti, F.S. 556 \\
\hline Urena lobata $\mathrm{L}$. & & $\mathrm{x}$ & Fernandes, A. (EAC 27821) \\
\hline Waltheria viscosissima A.St.-Hil. & & $\mathrm{x}$ & Fernandes, A. (EAC 4004) \\
\hline Wissadula periplocifolia (L.) C.Presl. ex Thwaites & & $\mathrm{x}$ & Fernandes, A. (EAC 2750) \\
\hline \multicolumn{4}{|l|}{ 46. Marantaceae } \\
\hline Goeppertia effusa Saka \& Lombradi & & $\mathrm{x}$ & Fernandes, A. (EAC 27840) \\
\hline G. squarros (Anderss. \& Kennedy) Borchs. \& S.Suárez & & $\mathrm{x}$ & Fernandes et al. (EAC 27920) \\
\hline Maranta protracta Miq. & & $\mathrm{x}$ & Fernandes, A. (EAC 27774) \\
\hline \multicolumn{4}{|l|}{ 47. Melastomataceae } \\
\hline Acisanthera variabilis (Mart. \& Schrank) Triana. & & $\mathrm{x}$ & Fernandes, A. (EAC 27755) \\
\hline Clidemia biserrata DC. & & $\mathrm{x}$ & Loiola, M.I.B. 1491 \\
\hline C. debilis Crueg. & & $\mathrm{x}$ & Castro, A.S.F. 154 \\
\hline C. hirta (L.) D.Don & & $\mathrm{x}$ & Fernandes, A. (EAC 27866) \\
\hline Miconia minutiflora (Bonpl.) DC. & & $\mathrm{x}$ & Cavalcanti, F.S. (EAC 28472) \\
\hline M. nervosa (Sm.) Triana & & $\mathrm{x}$ & Fernandes, A. (EAC 27834) \\
\hline M. prasina (Sw.) DC. & & $\mathrm{x}$ & Fernandes, A. (EAC 8211) \\
\hline \multicolumn{4}{|l|}{ 48. Meliaceae } \\
\hline Cedrela odorata $\mathrm{L}$. & & $\mathrm{x}$ & Matos (EAC 6897) \\
\hline Guarea macrophylla subsp. tuberculata (Vell.) T.D.Penn. & & $\mathrm{x}$ & Fernandes, A. (EAC 27775) \\
\hline Trichilia hirta L. & & $\mathrm{x}$ & Fernandes, A. (EAC 5009) \\
\hline \multicolumn{4}{|l|}{ 49. Molluginaceae } \\
\hline Mollugo verticillata $\mathrm{L}$. & & $\mathrm{x}$ & Fernandes, A. (EAC 27823) \\
\hline \multicolumn{4}{|l|}{ 50. Moraceae } \\
\hline Brosimum gaudichaudii Trécul & $\mathrm{x}$ & $\mathrm{x}$ & Fernandes, A. (EAC 27739) \\
\hline Ficus broadwayi Urb. & & $\mathrm{x}$ & Loiola, M.I.B. 1543 \\
\hline Maclura tinctoria (L.) D.Don ex Steud. & & $\mathrm{x}$ & Fernandes, A. (EAC 27838) \\
\hline \multicolumn{4}{|l|}{ 51. Myrsinaceae } \\
\hline Myrsineguianensis (Aubl.) Kuntze & & $\mathrm{x}$ & Fernandes, A. (EAC 27857) \\
\hline \multicolumn{4}{|l|}{ 52. Myrtaceae } \\
\hline Campomanesia aromatica (Aubl.) Griseb. & & $\mathrm{x}$ & Fernandes et al. (EAC 27806) \\
\hline Eugenia supraaxilaris Spring & $\mathrm{x}$ & & Sterile material \\
\hline E. dysenterica DC. & $\mathrm{x}$ & & Sterile material \\
\hline E. flavescens DC. & $\mathrm{x}$ & & Sterile material \\
\hline E. florida DC. & & $\mathrm{x}$ & Fernandes, A. (EAC 28012) \\
\hline E. pachnantha O.Berg & & $\mathrm{x}$ & Fernandes, A. (EAC 4011) \\
\hline Eugenia sp. & $\mathrm{x}$ & & Sterile material \\
\hline Myrcia multiflora (Lam.) DC & & $\mathrm{x}$ & Sterile material \\
\hline Myrcia sp.1 & & $\mathrm{x}$ & Fernandes, A. (EAC 27800) \\
\hline Myrcia sp. 2 & & $\mathrm{x}$ & Sterile material \\
\hline Psidium sp. & & $\mathrm{x}$ & Fernandes, A. (EAC 27502) \\
\hline 53. Nyctaginaceae & & & \\
\hline
\end{tabular}


Appendix A. Continued...

\begin{tabular}{|c|c|c|c|}
\hline \multirow{2}{*}{ FAMILY / SPECIES } & \multicolumn{2}{|c|}{ VEGETATION } & \multirow{2}{*}{ MAIN COLLECTOR } \\
\hline & TSDF & TSEF & \\
\hline Guapira graciliflora (Mart. ex Schmidt) Lundell & & $\mathrm{x}$ & Fernandes, A. et al. (EAC 28018) \\
\hline G. opposita (Vell.) Reitz & $\mathrm{x}$ & & Sterile material \\
\hline \multicolumn{4}{|l|}{ 54. Ochnaceae } \\
\hline Ouratea salicifolia (A.St.-Hil. \& Tul.) Engl. & & $\mathrm{x}$ & Fernandes, A. (EAC 5010) \\
\hline Sauvagesia erecta $\mathrm{L}$. & & $\mathrm{x}$ & Fernandes, A. (EAC 27776) \\
\hline \multicolumn{4}{|l|}{ 55. Olacaceae } \\
\hline Cathedra rubricaulis Miers & & $\mathrm{x}$ & Fernandes, A. (EAC 5025) \\
\hline \multicolumn{4}{|l|}{ 56. Opiliaceae } \\
\hline Agonandra brasiliensis Miers ex Benth. \& Hook.f. & $\mathrm{x}$ & $\mathrm{x}$ & Fernandes, A. (EAC 27758) \\
\hline \multicolumn{4}{|l|}{ 57. Orchidaceae } \\
\hline Oeceoclades maculata (Lind.) Lindl. & & $\mathrm{x}$ & Loiola, M.I.B. 1538 \\
\hline Polystachya concreta (Jacq.) Garay \& Sweet & & $\mathrm{x}$ & Fernandes, A. (EAC 27905) \\
\hline \multicolumn{4}{|l|}{ 58. Oxalidaceae } \\
\hline Oxalis alstonii Lourteig & & $\mathrm{x}$ & Loiola, M.I.B. 2197 \\
\hline O. cratensis Oliv. ex Hook. & & $\mathrm{x}$ & Loiola, M.I.B. 1496 \\
\hline O. grisea A.St.-Hil. \& Naudin & & $\mathrm{x}$ & Loiola, M.I.B. 1539 \\
\hline O. triangularis A.St.-Hil. & & $\mathrm{x}$ & Loiola, M.I.B. 1507 \\
\hline \multicolumn{4}{|l|}{ 59. Passifloraceae } \\
\hline Passiflora cincinnata Mast. & & $\mathrm{x}$ & Fernandes, A. (EAC 27757) \\
\hline P. glandulosa Cav. & & $\mathrm{x}$ & Castro,A.S.F. 1653 \\
\hline P. laurifolia L. & & $\mathrm{x}$ & Fernandes, A. (EAC 27825) \\
\hline P. misera Kunth & & $\mathrm{x}$ & Sterile material \\
\hline P. suberosa L. & & $\mathrm{x}$ & Sterile material \\
\hline P. tricuspis Mast. & & $\mathrm{x}$ & Sterile material \\
\hline Passiflora sp. & & $\mathrm{x}$ & Sterile material \\
\hline \multicolumn{4}{|l|}{ 60. Phyllanthaceae } \\
\hline Phyllanthus subermaginatus Mull.Arg. & & $\mathrm{x}$ & Castro, A.S.F. 1654 \\
\hline P. tenellus Roxb. & & $\mathrm{x}$ & Loiola, M.I.B. 1498 \\
\hline \multicolumn{4}{|l|}{ 61. Phytolaccaceae } \\
\hline Gallesia integrifolia (Spreng.) Harms & & $\mathrm{x}$ & Fernandes, A. (EAC 8668) \\
\hline Microtea glochidiata Moq. & & $\mathrm{x}$ & Fernandes, A. (EAC 27859) \\
\hline \multicolumn{4}{|l|}{ 62. Piperaceae } \\
\hline Peperomia circinnata Link & & $\mathrm{x}$ & Loiola, M.I.B. 1844 \\
\hline Piper arboreum Aubl. & $\mathrm{x}$ & $\mathrm{x}$ & Fernandes, A. (EAC 27753) \\
\hline P. caldense C.DC. & & $\mathrm{x}$ & Fernandes, A. (EAC 27727) \\
\hline P. divaricatum G.Mey. & & $\mathrm{x}$ & Fernandes, A. (EAC 27939) \\
\hline P. hispidum Sw. & & $\mathrm{x}$ & Fernandes, A. (EAC 27731) \\
\hline P. tuberculatum Jacq. & & $\mathrm{x}$ & Cavalcanti, F.S. (EAC 28461) \\
\hline \multicolumn{4}{|l|}{ 63. Plantaginaceae } \\
\hline Scoparia dulcis L. & & $\mathrm{x}$ & Loiola, M.I.B. 1526 \\
\hline \multicolumn{4}{|l|}{ 64. Plumbaginaceae } \\
\hline Plumbago scandens L. & & $\mathrm{x}$ & Cavalcanti, F.S. (EAC 28466) \\
\hline \multicolumn{4}{|l|}{ 65. Poaceae } \\
\hline Homolepis isocalycia (G.Mey.) Chase & & $\mathrm{x}$ & Fernandes, A. (EAC 28221) \\
\hline Lasiacis ligulata Hitchc. \& Chase & & $\mathrm{x}$ & Fernandes, A. (EAC 3912) \\
\hline Megathyrsus maximus (Jacq.) B.K.Simon \& S.W.L.Jacobs & & $\mathrm{x}$ & Fernandes, A. (EAC 27804) \\
\hline Olyra latifolia L. & & $\mathrm{x}$ & Fernandes, A. (EAC 27895) \\
\hline Oplismenus hirtellus (L.) P.Beauv. & & $\mathrm{x}$ & Loiola, M.I.B. 1796 \\
\hline Panicum millegrana Poir. & & $\mathrm{x}$ & Fernandes, A. (EAC 4225) \\
\hline Setaria adhaerens (Forssk.) Chiov. & & $\mathrm{x}$ & Fernandes, A. (EAC 27911) \\
\hline \multicolumn{4}{|l|}{ 66. Podostemaceae } \\
\hline Apinagia gardneriana Tul. & & $\mathrm{x}$ & Fernandes, A. (EAC 8671) \\
\hline
\end{tabular}


Appendix A. Continued...

\section{FAMILY / SPECIES}

VEGETATION TSDF

\section{MAIN COLLECTOR}

Bezerra, P. (EAC 5095)

Fernandes, A. (EAC 3890)

Martins, P. (EAC 8238)

Polygala sp.

68. Polygonaceae

Coccoloba mollis Casar

Triplaris gardneriana Wedd.

69. Portulacaceae

Talinum triangulare(Jacq.) Willd.

70. Ranunculaceae

Clematis affine A.St.-Hil.

71. Rhamnaceae

Gouania virgata Reissek

Ziziphus undulata Reissek

72. Rubiaceae

Bertiera guianensis Aubl.

Borreria verticillata (L.) G.Mey.

Chomelia pohliana Müll.Arg.

Coussarea hydrangeifolia (Benth.) Müll.Arg.

Coutarea hexandra (Jacq.) K. Schum.

Geophila repens (L.) I.M.Johnst.

Guettarda viburnoides Cham. \& Schltdl.

Ixora brevifolia Benth.

Palicourea marcgravii A.St.-Hil.

Psychotria bahiensis DC.

P. capitata Ruiz \& Pav.

P. carthagenensis Jacq.

P. colorata (Willd. ex Schult.) Müll.Arg.

P. deflexa DC.

Psychotria sp.

Randia armata (Sw.) DC.

Rudgea sp.

Sabicea cinerea Aubl.

73. Rutaceae

Ertela trifolia (L.) Kuntze

Esenbeckia grandiflora Mart.

Zanthoxylum caribaeum Lam.

Z. rhoifolium Lam.

\section{Salicaceae}

Banara guianensis Aubl.

Casearia commersoniana Cambess.

C. decandra Jacq.

C. grandiflora Cambess.

C. javitensis Kunth

Xylosma ciliatifolia (Clos) Eichler

75. Santalaceae

Phoradendron piperoides (Kunth) Trel.

76. Sapindaceae

Allophylus edulis (A.St.-Hil. et al.) Hieron. ex Niederl.

A. puberulus Radlk

Cardiospermum corindum L.

Cupania impressinervia Acev.-Rodr. $\mathrm{x}$

$\mathrm{x}$

$\mathrm{x}$
Loiola, M.I.B. 1924

Loiola, M.I.B. 2059 $\mathrm{x}$

$\mathrm{x}$

$\mathrm{x}$
Fernandes, A. (EAC 27950)

Fernandes, A.P. (EAC 15141)

Loiola, M.I.B. 1546

Fernandes, A. (EAC 27499)

Loiola, M.I.B. 1480

E.B. Souza

Brandão, E.K.S. 56

Souza, E.B. 2042

Sterile material

Loiola, M.I.B. 1786

Loiola, M.I.B. 2205

Fernandes, A. (EAC 3943)

Loiola, M.I.B. 1479

Loiola, M.I.B. 1478

Loiola, M.I.B. 1783

Loiola, M.I.B. 1789

Loiola, M.I.B. 1482

Loiola, M.I.B. 1781

Sterile material

Loiola, M.I.B. 1847

Fernandes, A. (EAC 28000)

Fernandes, A. (EAC 4215)

Souza, E.B. 3974

Cavalcanti, F.S. (EAC 28460)

Fernandes, A. (EAC 27752)

Loiola, M.I.B. 2239

Loiola, M.I.B. 2232

Fernandes, A. (EAC 27685)

Loiola, M.I.B. 2208

Fernandes, A. (EAC 27744)

Loiola, M.I.B. (EAC 53993)

$\mathrm{x}$

Lima-Verde, L.W. (EAC 27836)

$\mathrm{x}$

Fernandes, A. (EAC 27833)

$\mathrm{x}$

Loiola, M.I.B. 1474

Fernandes, A. (EAC 27917)

Castro, A.J. (EAC 6149)

Fernandes, A. (EAC 5004) 
Appendix A. Continued...

\begin{tabular}{|c|c|c|c|}
\hline \multirow{2}{*}{ FAMILY / SPECIES } & \multicolumn{2}{|c|}{ VEGETATION } & \multirow{2}{*}{ MAIN COLLECTOR } \\
\hline & TSDF & TSEF & \\
\hline C. oblongifolia Mart. & & $\mathrm{x}$ & Sterile material \\
\hline Matayba guianensis Aubl. & & $\mathrm{x}$ & Fernandes, A. (EAC 27742) \\
\hline Paullinia cearensis Somner \& Ferrucci & & $\mathrm{x}$ & Fernandes, A. (EAC 27799) \\
\hline P. pinnata L. & & $\mathrm{x}$ & Fernandes, A. (EAC 27796) \\
\hline Paullinia sp. & $\mathrm{x}$ & & Sterile material \\
\hline Serjania glabrata Kunth & & $\mathrm{x}$ & Loiola, M.I.B. 1821 \\
\hline S. hebecarpa Benth. & & $\mathrm{x}$ & Fernandes, A. (EAC 3928) \\
\hline Serjania sp. & $\mathrm{x}$ & $\mathrm{x}$ & Sterile material \\
\hline Talisia esculenta (Cambess.) Radlk. & $\mathrm{x}$ & $\mathrm{x}$ & Loiola, M.I.B. 1920 \\
\hline Urvillea laevis Radlk. & & $\mathrm{x}$ & Fernandes, A. (EAC 15135) \\
\hline \multicolumn{4}{|l|}{ 77. Sapotaceae } \\
\hline Chrysophyllum arenarium Allemão & & $\mathrm{x}$ & Cavalcanti, F.S. 530 \\
\hline C. rufum Mart. & & $\mathrm{x}$ & Fernandes, A. (EAC 7950) \\
\hline Pouteria macrophylla (Lam.) Eyma & $\mathrm{x}$ & $\mathrm{x}$ & Loiola, M.I.B. 2210 \\
\hline \multicolumn{4}{|l|}{ 78. Simaroubaceae } \\
\hline Simarouba amara Aubl. & & $\mathrm{x}$ & Fernandes, A. (EAC 27737) \\
\hline \multicolumn{4}{|l|}{ 79. Smilacaceae } \\
\hline Smilax irrorata Mart. ex Griseb & & $\mathrm{x}$ & Loiola, M.I.B. 2212 \\
\hline \multicolumn{4}{|l|}{ 80. Solanaceae } \\
\hline Acnistus arborescens (L.) Schltdl. & & $\mathrm{x}$ & Nunes, E. (EAC 27789) \\
\hline Capsicum parvifolium Sendtn. & & $\mathrm{x}$ & Fernandes, A. (EAC 27826) \\
\hline Cestrum axillare Vell. & & $\mathrm{x}$ & Mata, M.F. (EAC 15617) \\
\hline C. latifolium Lam. & & $\mathrm{x}$ & Fernandes, A. (EAC 5081) \\
\hline Schwenckia grandiflora Benth. & & $\mathrm{x}$ & Fernandes, A. (EAC 3968) \\
\hline Solanum asperum Rich. & & $\mathrm{x}$ & Loiola, M.I.B. 2237 \\
\hline S. campaniforme Roem. \& Schult. & & $\mathrm{x}$ & Fernandes, A. (EAC 27843) \\
\hline S. robustum H.L.Wendl. & & $\mathrm{x}$ & Fernandes, A. (EAC 27957) \\
\hline S. stipulaceum Willd. ex Roem. \& Schult. & & $\mathrm{x}$ & Fernandes, A. (EAC 27870) \\
\hline \multicolumn{4}{|l|}{ 81. Urticaceae } \\
\hline Cecropia pachystachya Trécul & & $\mathrm{x}$ & Cavalcanti, F.S. 533 \\
\hline Urera caracasana (Jacq.) Gaudich. ex Griseb. & $\mathrm{x}$ & & Carauta, J.P.P. 550 \\
\hline \multicolumn{4}{|l|}{ 82. Verbenaceae } \\
\hline Citharexylum sp. & & $\mathrm{x}$ & Fernandes, A. (EAC 6840) \\
\hline Lantana canescens Kunth & & $\mathrm{x}$ & Fernandes, A. (EAC 4005) \\
\hline L. fucata Lindl. & & $\mathrm{x}$ & Fernandes, A. (EAC 7939) \\
\hline Stachytarpheta cayenensis (Rich.) Vahl. & & $\mathrm{x}$ & Fernandes, A. (EAC 3999) \\
\hline \multicolumn{4}{|l|}{ 83. Violaceae } \\
\hline Pombalia communis (A.St.-Hil.) Paula-Souza & & $\mathrm{x}$ & Fernandes, A. (EAC 5655) \\
\hline \multicolumn{4}{|l|}{ 84. Vitaceae } \\
\hline Cissus gongylodes (Baker) Planch. & $\mathrm{x}$ & & Loiola, M.I.B. 1593 \\
\hline Clematicissus simsiana (Schult. \& Schult.f.) Lombardi & $\mathrm{x}$ & $\mathrm{x}$ & Fernandes, A. (EAC 27929) \\
\hline
\end{tabular}


Appendix B. List of alien species of the Tropical Seasonal Evergreen Forest (TSEF) and the Tropical Seasonal Deciduous Forest (TSDF) of the Ubajara National Park in Ceará, Brazil, where VN = vernacular name; GF = Growth form: tree (tr), shrub (sh), climber (cl), terrestrial herb (th); $\mathrm{O}=$ outskirts, $\mathrm{I}=$ inside, $\mathrm{x}=$ presence of taxa, ${ }^{\star}$ absence of vernacular name.

\begin{tabular}{|c|c|c|c|c|c|c|}
\hline \multirow{2}{*}{ FAMÍLIA/ESPÉCIE } & \multirow{2}{*}{$\mathbf{V N}$} & \multirow{2}{*}{ GF } & \multicolumn{2}{|c|}{ TSEF } & \multicolumn{2}{|c|}{ TSDF } \\
\hline & & & $\mathbf{O}$ & I & $\mathbf{O}$ & I \\
\hline \multicolumn{7}{|l|}{ 1. Amaryllidaceae } \\
\hline Crinum procerum Carey ex Herb. & crino-branco & th & $\mathrm{x}$ & & & \\
\hline Eucharis grandiflora Planch. \& Linden & estrela-d'alva & th & $\mathrm{x}$ & & & \\
\hline Hymenocallis littoralis (Jacq.) Salisb & lírio-aranha & th & $\mathrm{x}$ & & & \\
\hline \multicolumn{7}{|l|}{ 2. Anacardiaceae } \\
\hline Mangifera indica L. & mangueira & $\operatorname{tr}$ & $\mathrm{x}$ & $\mathrm{x}$ & $\mathrm{x}$ & $\mathrm{x}$ \\
\hline \multicolumn{7}{|l|}{ 3. Annonaceae } \\
\hline Annona squamosa $\mathrm{L}$. & ata & $\operatorname{tr}$ & $\mathrm{x}$ & $\mathrm{x}$ & $\mathrm{x}$ & $\mathrm{x}$ \\
\hline \multicolumn{7}{|l|}{ 4. Apocynaceae } \\
\hline Allamanda cathartica L. & alamanda & $\mathrm{cl}$ & $\mathrm{x}$ & & & \\
\hline Calotropis procera (Aiton) W.T.Aiton & algodão-da-praia & th & & & $\mathrm{x}$ & \\
\hline Cryptostegia madagascariensis Bojer ex Decne. & unha-do-diabo & th & & & $\mathrm{x}$ & $\mathrm{x}$ \\
\hline \multicolumn{7}{|l|}{ 5. Araceae } \\
\hline Aglaonema commutatum Schott & café-de-salão-dourado & th & $\mathrm{x}$ & & & \\
\hline Anthurium andraeanum L. & antúrio & th & $\mathrm{x}$ & & & \\
\hline Caladium $x$ hortulanum Birdsey & tinhorão & th & $\mathrm{x}$ & & & \\
\hline Dieffenbachia amoena Bull. & comigo-ninguém-pode & th & $\mathrm{x}$ & & & \\
\hline Philodendron imbe Schott ex Endl & $*$ & th & $\mathrm{x}$ & & & \\
\hline Spathiphyllum cannifolium (Dryand. ex Sims) Schott & * & th & $\mathrm{x}$ & & & \\
\hline \multicolumn{7}{|l|}{ 6. Arecaceae } \\
\hline Chamaedorea cataractarum Mart. & palmeira-cascata & $\operatorname{tr}$ & $\mathrm{x}$ & & & \\
\hline \multicolumn{7}{|l|}{ 7. Balsaminaceae } \\
\hline Impatiens walleriana Hook.f. & maria-sem-vergonha & th & $\mathrm{x}$ & & & \\
\hline \multicolumn{7}{|l|}{ 8. Davalliaceae } \\
\hline Nephrolepis biserrata (Sw.) Schott & rabo-de-peixe & th & $\mathrm{x}$ & & & \\
\hline N. pectinata (Willd.) Schott & samambaia & th & $\mathrm{x}$ & & & \\
\hline \multicolumn{7}{|l|}{ 9. Heliconiaceae } \\
\hline Heliconia bihai $\mathrm{L}$. & $*$ & $\operatorname{tr}$ & $\mathrm{x}$ & & & \\
\hline H. psittacorum L. & helicônia, bananinha & $\operatorname{tr}$ & $\mathrm{x}$ & & & \\
\hline \multicolumn{7}{|l|}{ 10. Iridaceae } \\
\hline Neomarica candida (Hassl.) Sprague & falso-íris & th & $\mathrm{x}$ & & & \\
\hline \multicolumn{7}{|l|}{ 11. Melastomataceae } \\
\hline Tibouchina sp. & quaresmeira & sh & $\mathrm{x}$ & & & \\
\hline \multicolumn{7}{|l|}{ 12. Meliaceae } \\
\hline Azadirachta indica A. Juss & $\operatorname{nim}$ & $\operatorname{tr}$ & & & $\mathrm{x}$ & \\
\hline \multicolumn{7}{|l|}{ 13. Moraceae } \\
\hline Artocarpus heterophyllus Lam. & jaqueira & $\operatorname{tr}$ & $\mathrm{x}$ & $\mathrm{x}$ & & \\
\hline \multicolumn{7}{|l|}{ 14. Poaceae } \\
\hline Bambusa sp. & bambu & $\operatorname{tr}$ & $\mathrm{x}$ & $\mathrm{x}$ & & \\
\hline \multicolumn{7}{|l|}{ 15. Rubiaceae } \\
\hline Coffea arabica $\mathrm{L}$. & café & sh & & $\mathrm{x}$ & & \\
\hline \multicolumn{7}{|l|}{ 16. Rutaceae } \\
\hline Citrus latifolia Tan. & limoeiro & $\operatorname{tr}$ & & & $\mathrm{x}$ & \\
\hline \multicolumn{7}{|l|}{ 17. Zingiberaceae } \\
\hline Alpinia purpurata (Vieill.) K.Schum. & * & $\operatorname{sh}$ & $\mathrm{x}$ & & & \\
\hline
\end{tabular}

